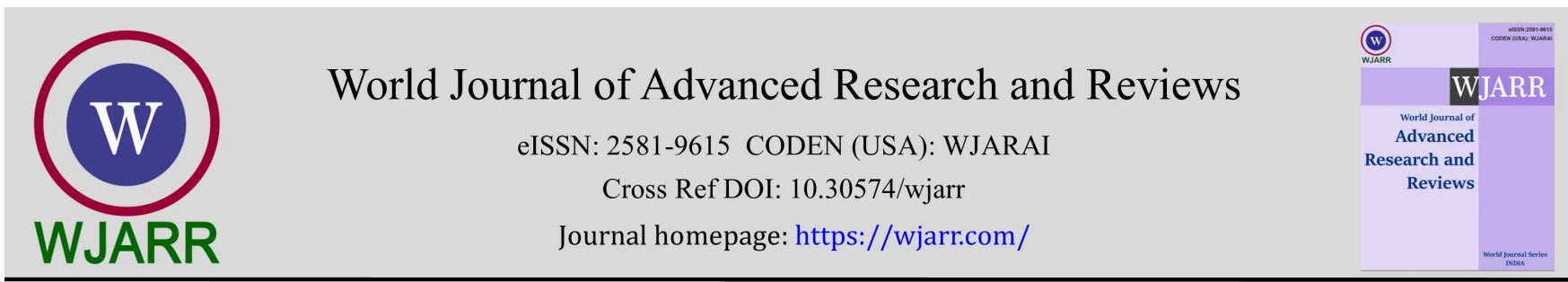

(RESEARCh ARticle)

\title{
Evaluation of in vitro dissolution similarity of bisoprolol film-coated tablets based on Weibull modelling using MATLAB ${ }^{\mathrm{TM}}$ simulation software
}

\author{
Ivana Mitrevska ${ }^{1,{ }^{*}}$, Hristijan Mickoski ${ }^{2}$, Katerina Brezovska ${ }^{3}$ and Aneta Dimitrovska ${ }^{3}$ \\ ${ }^{1}$ Research \& Development, Alkaloid AD, Skopje, R. North Macedonia. \\ ${ }^{2}$ Institute of Mechanics, Ss. Cyril and Methodius University, Faculty of Mechanical Engineering, Skopje, R. North \\ Macedonia. \\ ${ }^{3}$ Institute of Applied Chemistry and Pharmaceutical Analysis, Ss. Cyril and Methodius University, Faculty of Pharmacy, \\ Skopje, R. North Macedonia.
}

World Journal of Advanced Research and Reviews, 2021, 10(01), 056-073

Publication history: Received on 01 March 2021; revised on 02 April 2021; accepted on 04 April 2021

Article DOI: https://doi.org/10.30574/wjarr.2021.10.1.0143

\begin{abstract}
The aim of this study was to compare the in vitro dissolution behaviour of reference (R) and generic-test (T) medicinal products with non-linear effects model. Mathematical function Weibull, was employed as basis for the non-linear effects model, coupled with MATLABTM simulation software to describe the release profile of the active substance. Medicinal products selected for the presented study include immediate-release tablets Concor $10 \mathrm{mg}$ and Bisoprolol $10 \mathrm{mg}$, which belongs to BCS class 1 of biopharmaceutics classification system. The result from the study indicated that Weibull distribution function coupled with computer-based program is more useful for comparison of the dissolution profiles. This combined approach provides robust and informative results, with accurate estimation on the in vitro performance for the medicinal products and it's the most suitable tool for prediction of in vivo behaviour of the medicinal product. In summary, we have employed Simulink graphical programming to design our system in a simulation environment.
\end{abstract}

Keywords: Dissolution profiles; Mathematical function; MATLAB ${ }^{\mathrm{TM}}$ simulation software; Simulink; Bisoprolol immediate-release tablets.

\section{Introduction}

Over the recent years, active substance release/dissolution from solid pharmaceutical dosage forms has been the subject of intensive and profitable scientific developments. Whenever a new solid dosage form is developed or produced, it is necessary to ensure that the dissolution of the active substance occurs in an appropriate manner [1]. The pharmaceutical industry and the regulatory authorities recently focus on active substance dissolution studies. There are many factors that influence the in vivo performance of the oral dosage form, and identification of these factors is crucial for developing generic medicinal product similar to reference product. The quantitative analysis of the values obtained in dissolution/release tests is facilitated when mathematical formulas expressing the dissolution results as a function of some of the dosage forms characteristics, are used. In some cases, these mathematic models are derived from the theoretical analysis of the process. In most of the cases, the theoretical concept does not exist and some empirical equations have proved to be more appropriate. The most of the model-dependent methods rely on a curve fitting procedure. Different mathematical functions have been used to model the observed data [2]. A distinction can be made between linear (zero-order, Higuchi, Hixson-Crowell, quadratic, polynomials) and non-linear models (first-order, Weibull, Korsmeyer-Peppas, Logistic, Gompertz). Some of these models like Hixson-Crowell and Higuchi, are derived from the theoretical concepts of the dissolution process. Since the latter can be complicated, it is often difficult to describe it mathematically in a correct way. Hence, empirical equations like the Weibull have proven to be more suitable.

\footnotetext{
* Corresponding author: Ivana Mitrevska

Research \& Development, Alkaloid AD, Skopje, R. North Macedonia
}

Copyright (C) 2021 Author(s) retain the copyright of this article. This article is published under the terms of the Creative Commons Attribution Liscense 4.0. 
The advantage of the use of mathematical models is in facilitation of the analysis and interpretation of the observed data because they describe the dissolution profiles as a function of only a few model parameters that can be statistically compared. A disadvantage is that these models are rather rigid and none of these models is suitable to fit all kinds of dissolution curves. Once a model is selected, the dissolution profiles are compared and evaluated in terms of the model parameters. In the meantime, the mathematical model approach also provides an insight into the mechanism of release of the active substance [3]. In general, the Weibull model, introduced by Langenbucher, has shown to be one of the most successful model in fitting the experimental dissolution curves to theoretical functions [4]. Additionally, the shape parameter in Weibull models is also important for describing the kinetic properties of the medicine [5]. The shape parameter for Weibull distribution provides vital information about the rate of release and helps to better understand the active substance release profile. Therefore, in vivo estimates have become very important with computer and simulation programs applied in the field of pharmacy [6].

In this paper, the performance of non-linear effects model was examined using the commercially available simulation software MATLAB ${ }^{\mathrm{TM}}$. The theoretical part, included to make the technique more accessible to non-statisticians. However, additional purpose was to show the ability of reducing the cost and ethically controversial animal and human experiments and to form biowaiver evaluations with these new approach in order to investigate if there are any in vitro differences between compared reference and generic medicinal products. Finally, a brief introduction to MATLAB ${ }^{\mathrm{TM}}$ programs for numerical simulation of random processes and procedures for maximum likelihood estimation are presented.

\section{Material and methods}

\subsection{Material and equipment}

Concor $10 \mathrm{mg}$ film-coated tablets were used as reference medicinal product and Bisoprolol $10 \mathrm{mg}$ film-coated tablets were used as generic-test medicinal product. Bisoprolol fumarate working standard (WS) was used as reference substance. Potassium dihydrogen phosphate and sodium hydroxide, used were with analytical grade.

The following instruments were used: six-station dissolution apparatus (Varian-Vankel 7025 Model: 115/230) complying with USP general methods, pH meter (Mettler Toledo), hotplate stirrer (IKA C-MAG HS7), analytical balance (Sartorius CPA 225D-OCE). The dissolution media: $\mathrm{pH} 1.2$ ( $\mathrm{HCl}, \mathrm{NaCl}$ ), acetate buffer $\mathrm{pH} 4.5$ and phosphate buffer $\mathrm{pH}$ 6.8 were prepared according to European Pharmacopeia. The amount of dissolved active substance was determined with HPLC method. Analysis were performed using the column Spherisorb Phenyl, $125 \mathrm{~mm} \times 4.0 \mathrm{~mm}, 5 \mu \mathrm{m}$, maintained at ambient temperature, with detection on $227 \mathrm{~nm}$. The mobile phase included mixture of buffer $\mathrm{pH} 3.0(2.0 \mathrm{~mL}$ Triethylamine in $1000 \mathrm{~mL}$ water) as solvent A and acetonitrile as solvent B in ratio, 80/20 ( $\mathrm{V} / \mathrm{V} \%)$, in isocratic mode. The flow rate was set to $1.2 \mathrm{~mL} / \mathrm{min}$. The analysis run time was 5 minutes and the injection volume was $20 \mu \mathrm{L}$ [7].

\subsection{In vitro release study}

The dissolution tests on film-coated tablets were carried out at $37 \pm 0.5^{\circ} \mathrm{C}$ and $75 \mathrm{r} / \mathrm{min}$ using qualified Apparatus II (paddle) with $900 \mathrm{~mL} \pm 1 \%$ of each medium. Sampling aliquots of $10 \mathrm{~mL}$ were withdrawn at 10, 15, 20 and 30 minutes and replaced with an equal volume of the fresh medium maintained at the same temperature [8]. After the end of each test time, samples aliquots were filtered through $0.20 \mu \mathrm{m}$ membrane filter (regenerated cellulose, RC), diluted with suitable amount of respective dissolution medium. Tests were performed with twelve (12) film-coated tablets and the obtained values are used for data analysis. A Bisoprolol tablet is official pharmacopoeial product (USP 42/NF 37) where buffer $\mathrm{pH} 1.2(\mathrm{HCl}, \mathrm{NaCl})$ is listed as a dissolution medium of choice for combined medicinal product with Hydrochlorothiazide [9]. Additionally, comparative dissolution testing in $\mathrm{pH} 4.5$ acetate buffer and pH $6.8 \mathrm{phosphate}$ buffer was performed. Samples obtained from dissolution experiments were quantitatively analysed using a HPLC method previously validated.

\subsection{Kinetic analysis of in vitro release profile}

Bisoprolol fumarate release from oral dosage form was studied using various mathematical models. Model-independent method (difference factor $f_{1}$ and similarity factor $f_{2}$ ) was not used, because this method is sensitive to the number of data points and these factors do not consider the correlation or variability of the data. ANOVA based methods also have their own deficiencies, especially in ignoring the correlation between the time points [10]. Although many mathematical models have been described to fit dissolution curves, only the most 'promising' ones were selected to be translated to NLE (non-linear effects) models [11]. As mentioned in the introduction, the dissolution process is difficult to describe pure theoretically and empirical functions give often better results. The model used for mathematical analysis in this paper can be broadly classified as statistical model, Weibull distribution function [12]. The Weibull distribution depends 
of two (2) parameters: $\beta$, is the shape parameter and $\alpha$, is the scale parameter that determines the released fraction of the active substance from the formulation. The mathematical expression for the Weibull distribution is as follow [13]:

$f(x)=1-e-\left(\frac{x}{\alpha}\right)^{\beta} ; x \geq 0$

where, function $f(x)$ is a released fraction of the active substance from the dosage form during the time $x$. The density of the probability distribution function ( $p d f)$ and is defined by the following function [14]:

$f(x)=\frac{\beta}{\alpha}\left(\frac{x}{\alpha}\right)^{\beta-1} e-\left(\frac{x}{\alpha}\right)^{\beta} ; x \geq 0$

Furthermore, the estimation of the scale and shape parameters $\alpha$ and $\beta$ can be provided with linear regression $y=a x+$ $b$, from which we get [15]:

$\ln \left[\ln \left(\frac{1}{1-F(x)}\right)\right]=\beta \ln x-\beta \ln \alpha$

Consequently, $y$-corresponds to the left part of the last equation, $x$-corresponds to $\ln (x)$, the parameter $\beta$ corresponds to the coefficient $a$, which gives the slope of the line to $\mathrm{x}$-axis (abscissa), and the expression $\beta \ln (\alpha)$ corresponds to the coefficient $b$ (intercept of the lines with the y-axis, ordinate). According to the above equations, the following functions is used in MATLAB ${ }^{\mathrm{TM}}$ : EXCEL $=$ beta $*(\mathrm{LN}(\operatorname{SLCIS} 0))^{\wedge}(1 /$ alpha) and [parmHat] = wblfit (x). Hence, sequence of random numbers was obtained for the given distribution parameters $\alpha$ and $\beta$ [16,17]. The shape parameter, $\beta$, characterizes the curve as either exponential $(\beta=1)$, sigmoid, $S$-shaped, with upward curvature followed by a turning point $(\beta>1)$, or parabolic, with a higher initial slope and after that consistent with the exponential $(\beta<1)$. The Weibull distribution is using in the calculation of the probability of dynamic processes such as dissolution of the dosage forms, and for the analysis of risks in processes of: aging if $\beta>1$, and exploitation at $\beta<1$ or $\beta=1$. The parameter $\beta$ in practice usually tends to value greater than 1, while $\alpha>0$ [18]. How the parameters $\alpha$ and $\beta$ affect the course of the dissolution profile is illustrated in Figure 1 [19].

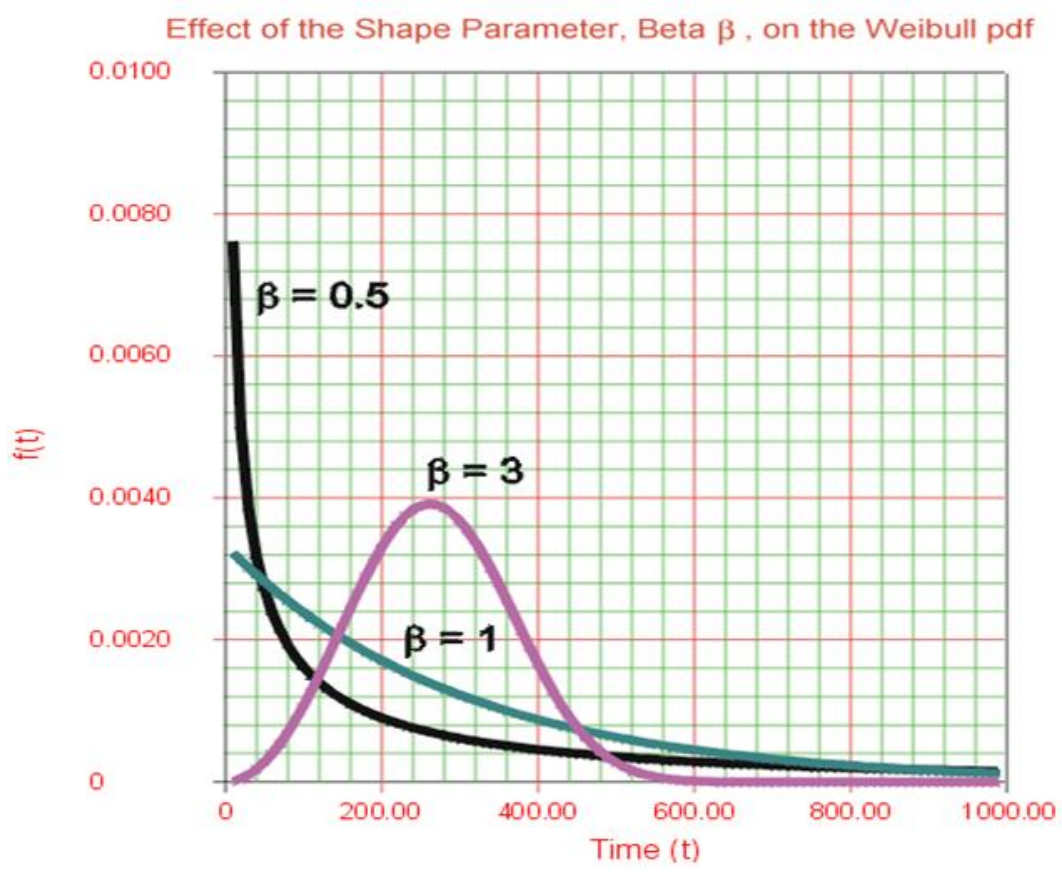

Figure 1 Two-parameter Weibull density plots.

\subsection{Computer program}


The programs were executed in MATLAB ${ }^{\mathrm{TM}}$ version 2021 that is general-purpose mathematical tool for simulation and modelling used in almost every branch of engineering environment. We present the "Statistics toolbox", a new software library that extends MATLAB ${ }^{\mathrm{TM}}$ and its support a robust and efficient analysis of complex datasets, affected by different sources of heterogeneity. Statistics and Machine Learning Toolbox ${ }^{\mathrm{TM}}$ provides functions and apps to describe, analyse and model data. Descriptive statistics, visualizations, data analysis, fit probability distributions to data and generated random numbers simulations, was carried out. Regression and classification algorithms allow us to draw conclusions from data and build predictive models. The software package "Statistics toolbox" is a set of programs that solve various problems in line with data analysis using methods of mathematical statistics. Most functions are implemented as $\mathrm{m}$ files. The "Statistics toolbox" contains more than $200 \mathrm{~m}$-files, where 20 basic distribution laws for continuous and discrete random quantities are applied. Thereby, seven functions for each of these distribution laws were provided to clarify: assessment of the parameters for law of distribution in accordance to experimental data; evaluation of distribution parameters through (fit) function; probability density distribution function, (pdf) and cumulative distribution function, (cdf). Moreover, MATLAB ${ }^{\mathrm{TM}}$ uses distribution-specific functions as: Weibull cumulative distribution function (wblcdf); Weibull probability density function (wblpdf), Weibull inverse cumulative distribution function (wblinv); Weibull mean and variance (wblstat); Weibull parameter estimates (wblfit), Weibull random numbers (wblrnd) and Weibull probability plot (wblplot) [19]. The program was used to facilitate the evaluation of similarity between dissolution data, to create a model data library that meets release rate data using non-linear optimization method and to compare dissolution profiles.

\section{Results and discussion}

The in vitro dissolution studies were performed at different $\mathrm{pH}$ conditions (pH 1.2, $\mathrm{pH} 4.5$ and $\mathrm{pH}$ 6.8). The first set of data in Figure 2a shows that both products dissolved very rapidly in $\mathrm{pH} 1.2$, more than $85 \%$ in 15 minutes. Moreover, in $\mathrm{pH} 4.5$ (Figure $2 \mathrm{~b}$ ) and pH 6.8 (Figure $2 \mathrm{c}$ ), T-product also dissolved very rapidly ( $>85 \%$ in 15 minutes), while the Rproduct, dissolved rapidly ( $>85 \%$ in 30 minutes).

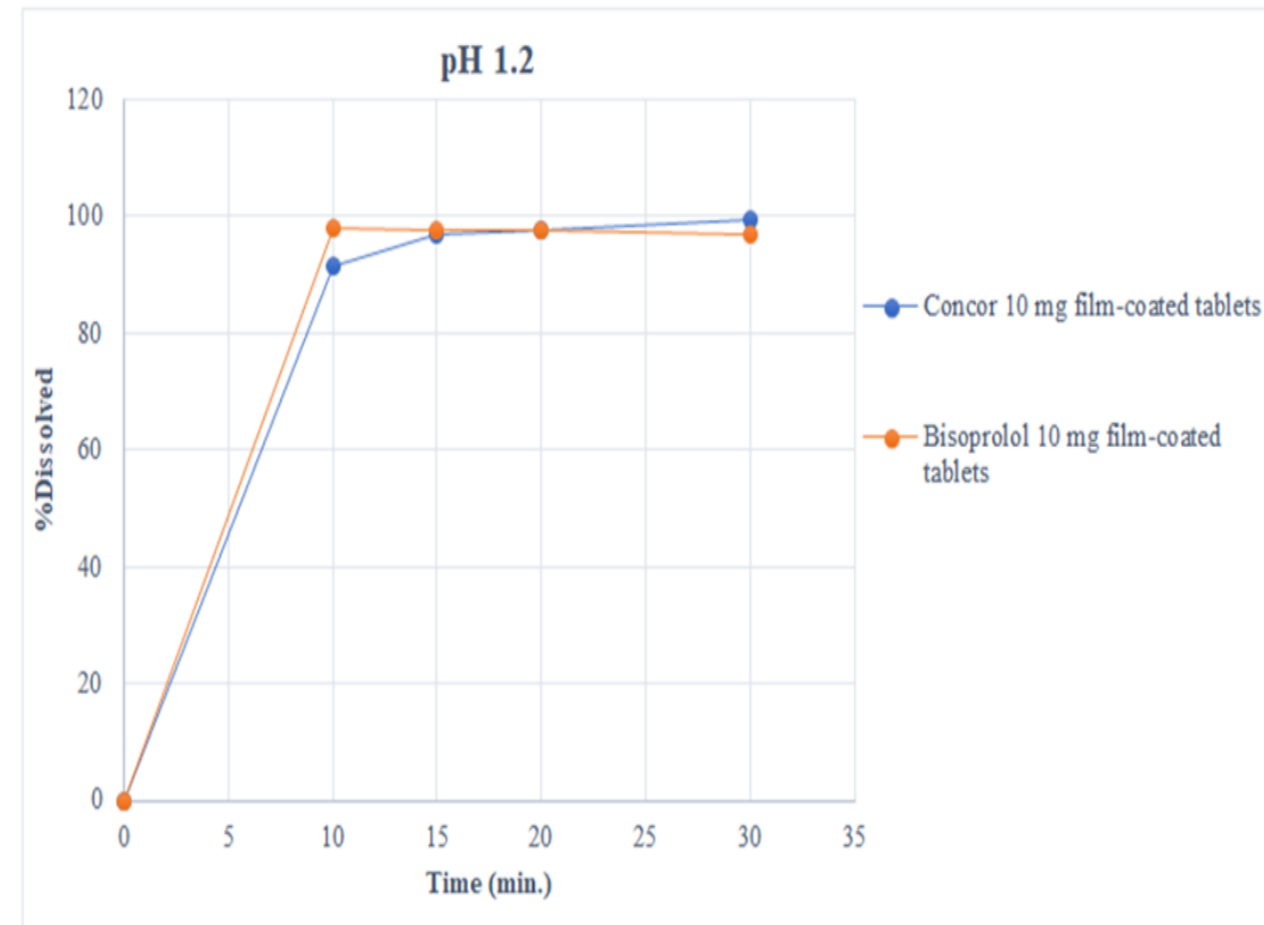

(a) 


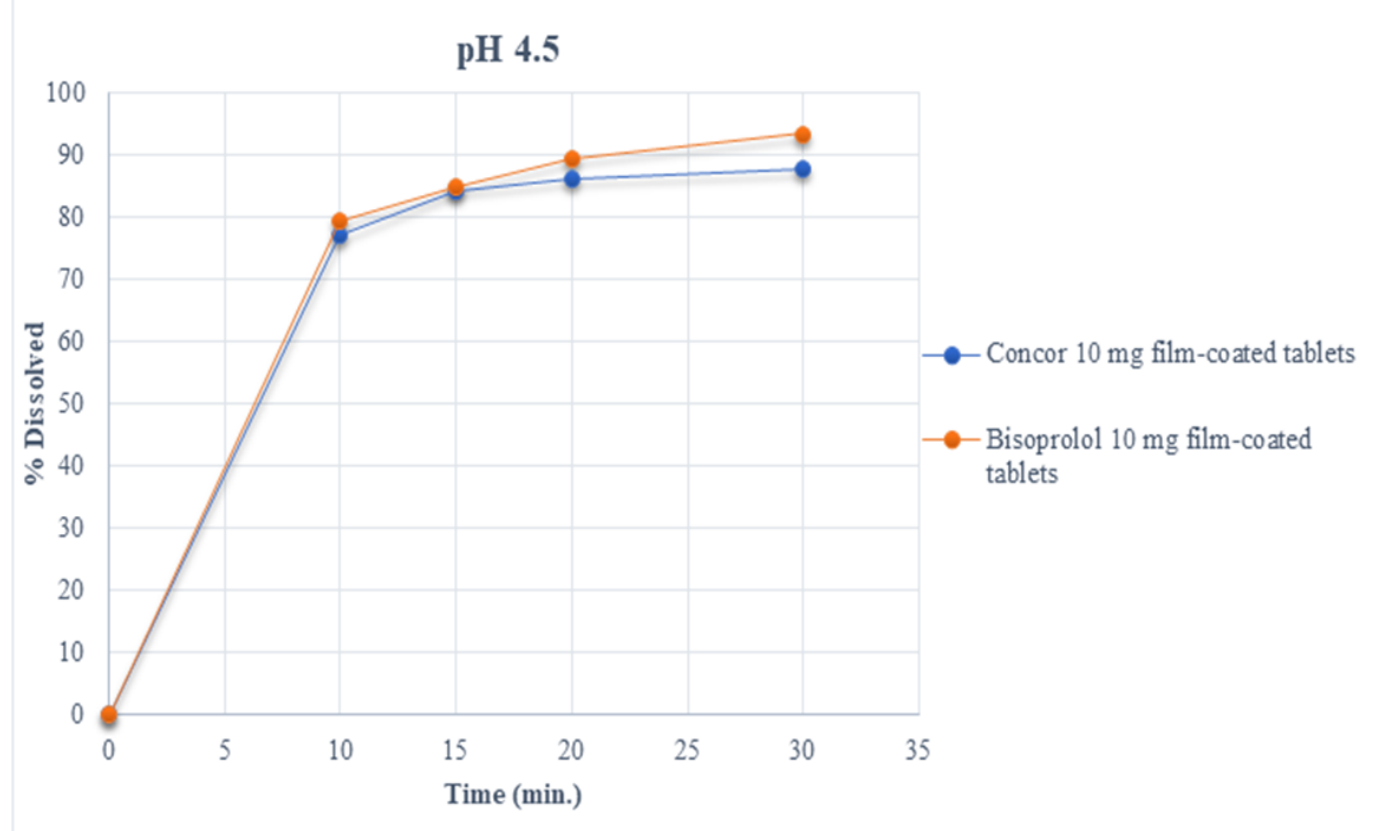

(b)

pH 6.8

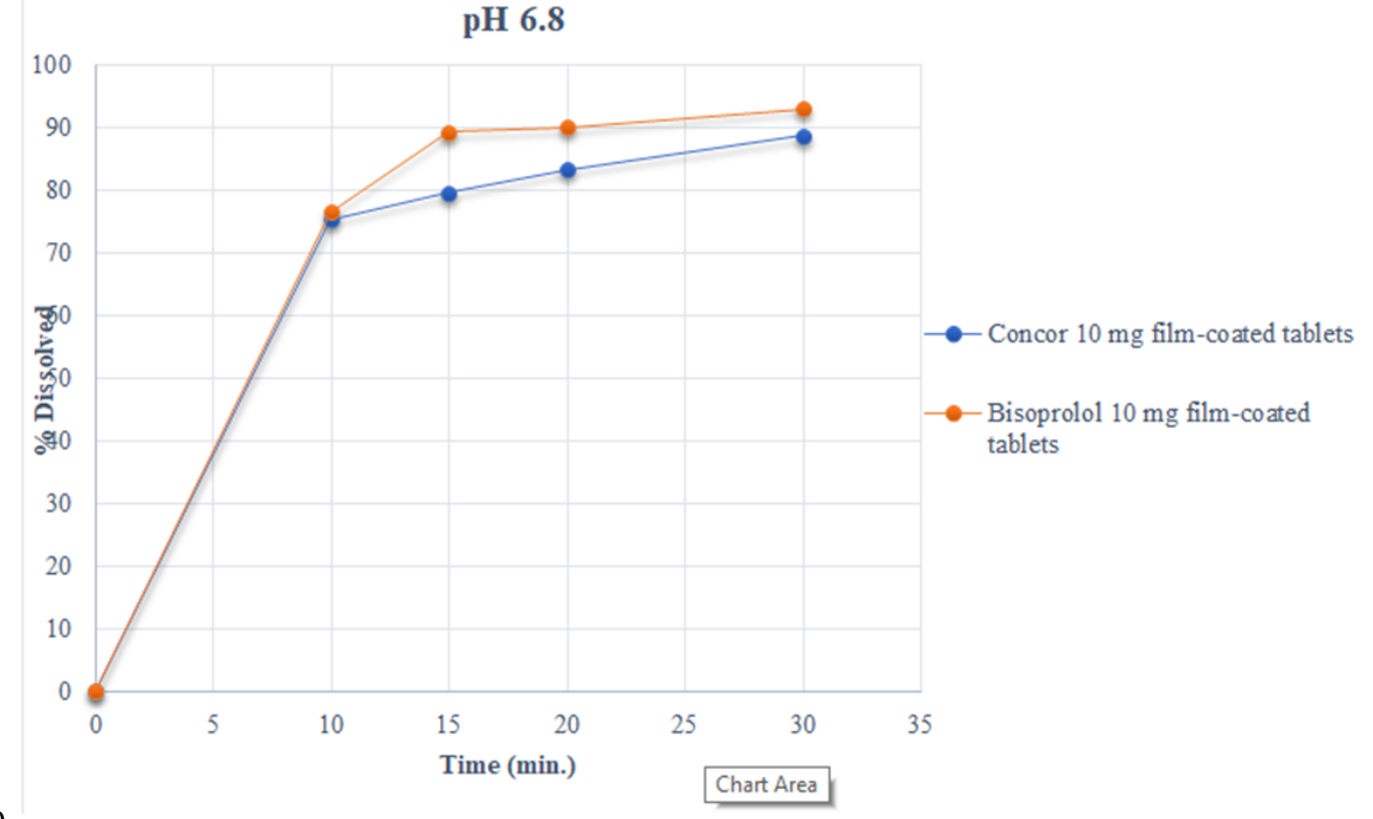

(c)

Figure 2 In vitro dissolution profiles in compendial media (a: $\mathrm{pH}$ 1.2, b: pH 4.5, c: pH 6.8).

Hence, the results obtained from the dissolution medium buffer $\mathrm{pH} 1.2$ are already relatively high therefore, further mathematical calculations are not needed and products can be considered similar to each other [21]. At pH 4.5 and pH 6.8 , for all generated profiles, more than one mean value above $85 \%$ for any of the tested batches was observed, creating a consequence that the $f_{2}$ statistics for determining profile similarity is not applicable. In accordance to the guideline $[20,21]$, in such cases alternative mathematical or statistical methodologies can be carried out for demonstrating dissolution similarity. The decision that two products are similar depends not only on the model chosen, but also on the significance used level. Nowadays, there are no agreements between the regulatory authorities and the pharmaceutical industry which level is pharmaceutically acceptable. However, compared to the standard $f_{2}$ factor, non-linear effects models are more discriminative and informative [22]. Dissolution kinetics were evaluated by model dependent method, Weibull distribution function coupled with MATLAB ${ }^{\mathrm{TM}}$ simulation software. The Weibull distribution is a two-parameter family of curves. This distribution is named for Waloddi Weibull, who offered it as an appropriate analytical tool for modelling [13]. Current usage also includes reliability and lifetime modelling. It must be emphasized that, in statistical systems, the presence of a maximum quantity for distribution, is particularly important when solving tasks for 
identifying distribution laws for samples. Therefore, in most statistical systems, calculation of the obtained data are realized with electronic spreadsheet editor. Hence, through performed analysis of the dissolution data obtained from the test and reference products, an overview of the "Statistics toolbox" algorithms was introduced.

The likelihood function is the probability density function (pdf) viewed as a function of the parameters. The maximum likelihood estimates (MLEs) are the parameter estimates that maximize the likelihood function for fixed values of $\mathrm{x}$. The maximum likelihood estimators of $\alpha$ and $\beta$ for the Weibull distribution are the solution of the simultaneous equations:

$\hat{a}=\left[\left(\frac{1}{n}\right) \sum_{i=1}^{n} x_{i}^{\hat{b}}\right] \frac{1}{\hat{b}}$

$\hat{b}=\frac{n}{\left(\frac{1}{\hat{a}}\right) \sum x_{i}^{\widehat{b}} \log x_{i}-\sum_{i-1}^{n} \log x_{i}}$

Where, $\hat{a}$ and $\hat{b}$ are unbiased estimators of the parameters $\alpha$ and $\beta$.

The values of the scale and shape parameters of the non-linear regressions are listed in Table 1.

Table 1 Estimated scale and shape parameters for tested batches.

\begin{tabular}{|c|c|c|}
\hline & Reference drug product- $R$ & Test drug product- $T$ \\
\hline \multicolumn{3}{|l|}{ pH 4.5} \\
\hline$\alpha$ - scale parameter & 0.000065 & 0.000132 \\
\hline$\beta$-shape parameter & 2.7 & 2.9 \\
\hline \multicolumn{3}{|l|}{ pH 6.8} \\
\hline$\alpha$-scale parameter & 0.00014 & 0.000145 \\
\hline$\beta$ - shape parameter & 2.7 & 2.9 \\
\hline
\end{tabular}

The shape, parameter, $\beta$, characterizes the curve as sigmoid, S-shaped, with upward curvature followed by a turning point $(\beta>1)$ for all tested batches. According to the linear relation (Eq.3), shape parameter $(\beta=a)$ was obtained from the slope of the line and the scale parameter, $\alpha$, was estimated from the ordinate value. Additionally, in Table 2 linear regressions of the estimates for $b$ and $a v s . \mathrm{n}$ (time points) are given.

Table 2 Estimated a and b coefficients for tested batches.

\begin{tabular}{|l|l|l|}
\hline \multicolumn{2}{|l|}{ Reference drug product-R } & Test drug product-T \\
\hline pH 4.5 & $y=2.724 x+26.256$ & $y=2.9031 x+25.936$ \\
\hline $\begin{array}{l}\text { Linear } \\
\text { regression }\end{array}$ & 2.724 & 2.9031 \\
\hline$a$ & 26.256 & 25.936 \\
\hline$b$ & $y=2.7391 x+24.314$ & $y=2.9265 x+25.869$ \\
\hline pH 6.8 & & 2.9265 \\
\hline $\begin{array}{l}\text { Linear } \\
\text { regression }\end{array}$ & 2.7391 & 25.869 \\
\hline$a$ & 24.314 & \\
\hline$b$ &
\end{tabular}


Furthermore, the Weibull probability density function was calculated by equation 6 .

$f(x \mid a, b)=\frac{b}{a}\left(\frac{x}{a}\right)^{b-1} e-\left(\frac{x}{a}\right)^{b} ; x \geq 0$

Assessment with $m$-file in MATLAB ${ }^{\mathrm{TM}}$ was obtained as follows (Figures 3 and 4):

$\mathrm{x}=0: 1: 30 ; \mathrm{y}=\operatorname{wblpdf}(\mathrm{x}, a, b) ; \mathrm{x}=0: .1: 30 ; \mathrm{y}=\operatorname{pdf}(\mathrm{pd}, \mathrm{x}) ; \operatorname{plot}\left(\mathrm{x}, \mathrm{y},{ }^{\prime}\right.$ LineWidth' $\left.^{\prime} 2\right)$

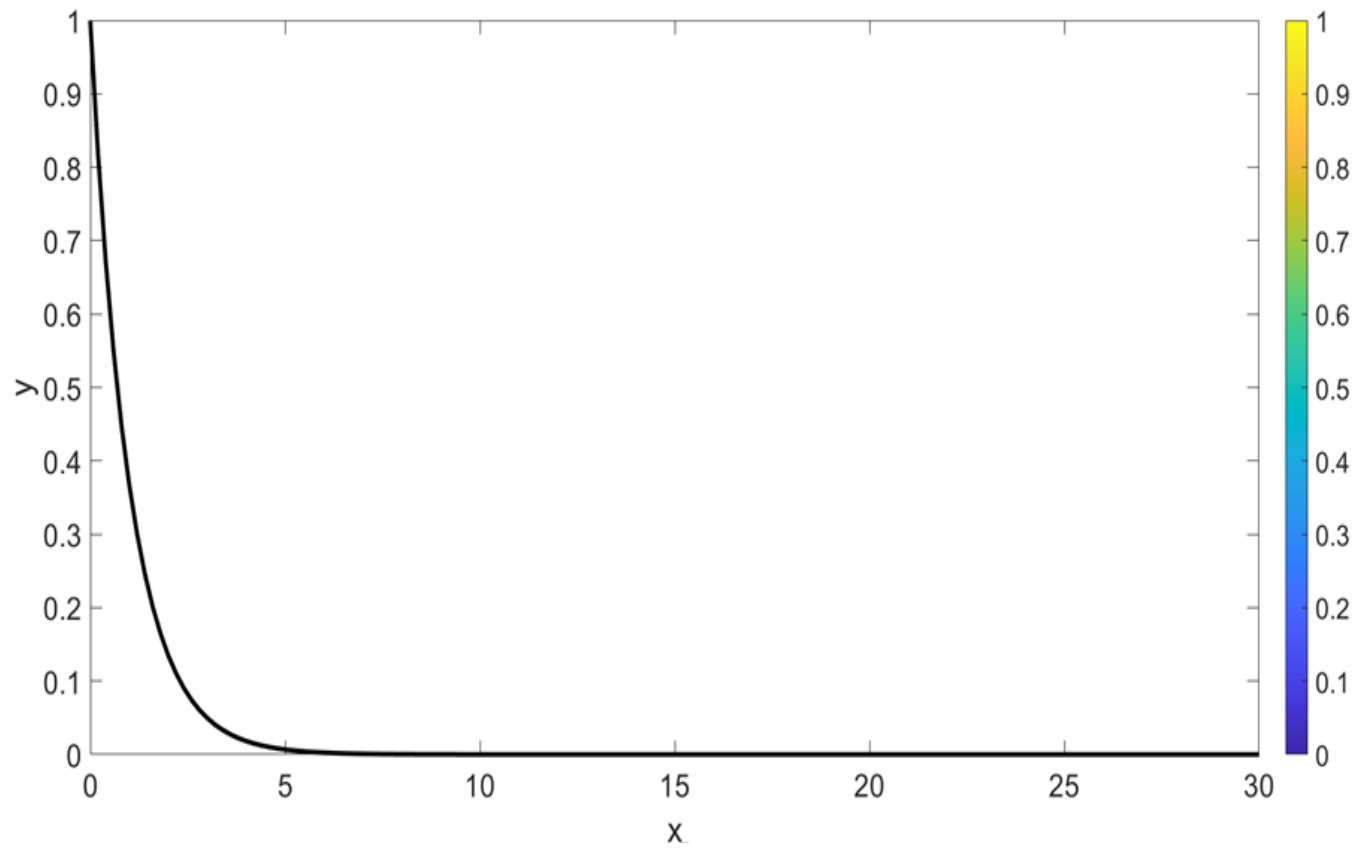

(a)

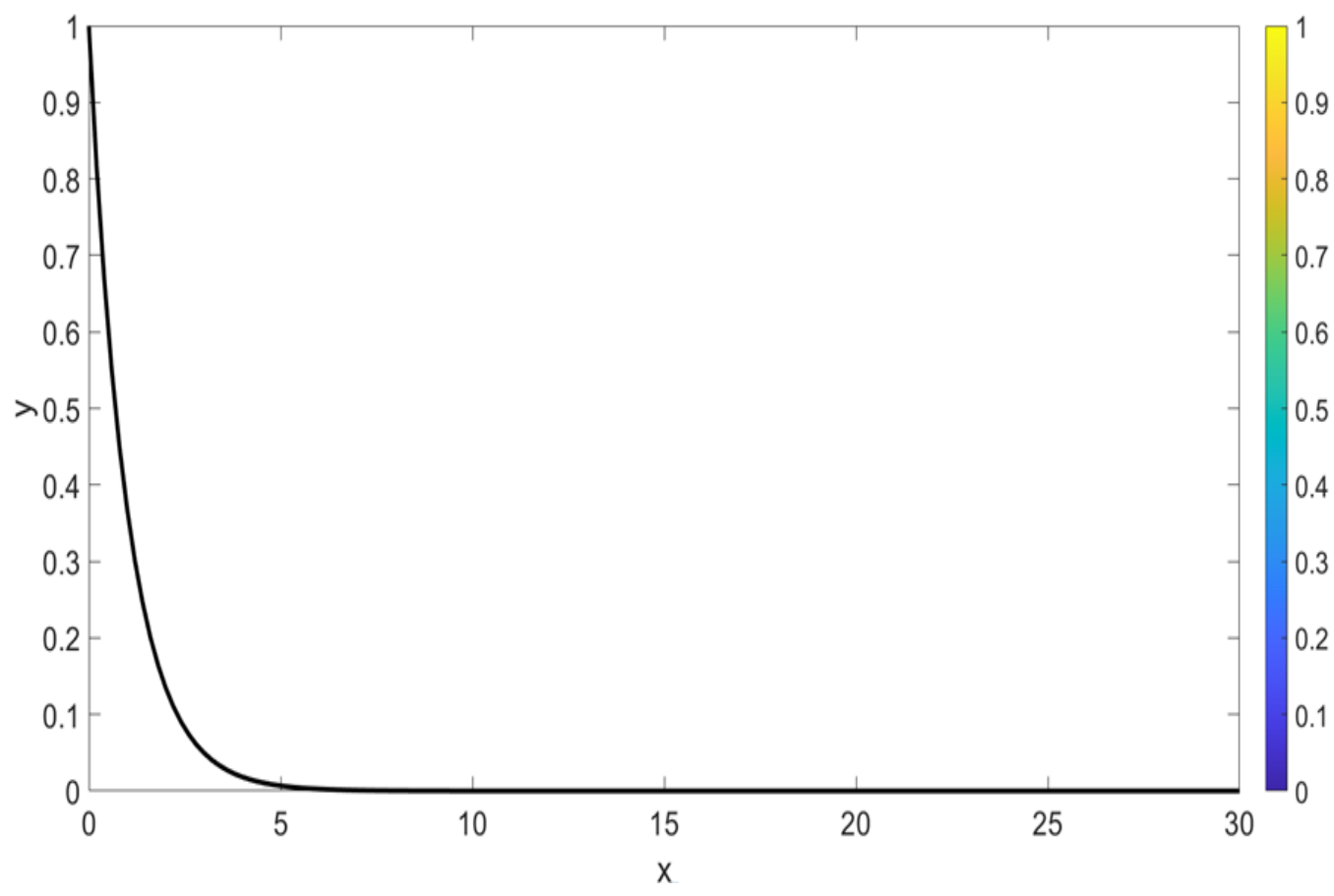

Figure 3 Weibull probability density function for Reference product (a: $\mathrm{pH} 4.5, \mathrm{~b}: \mathrm{pH} 6.8$ ). 


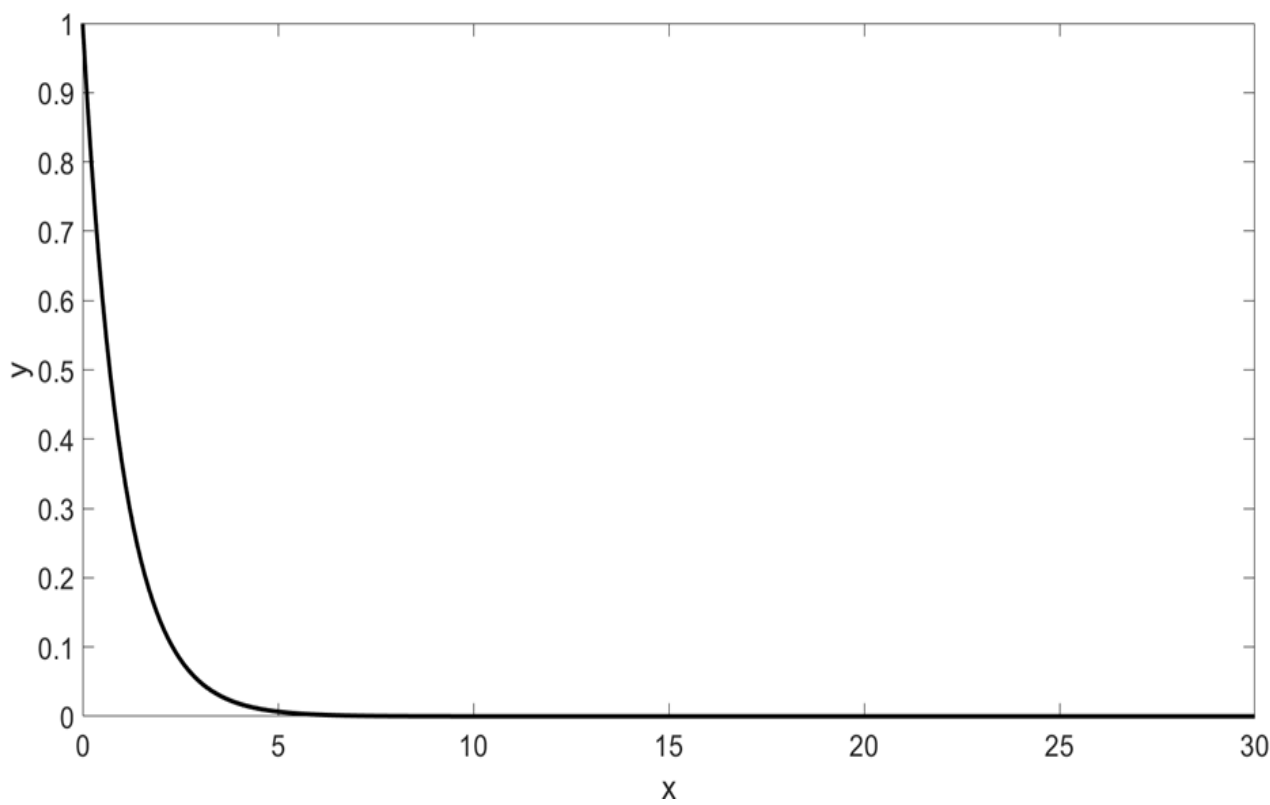

(a)

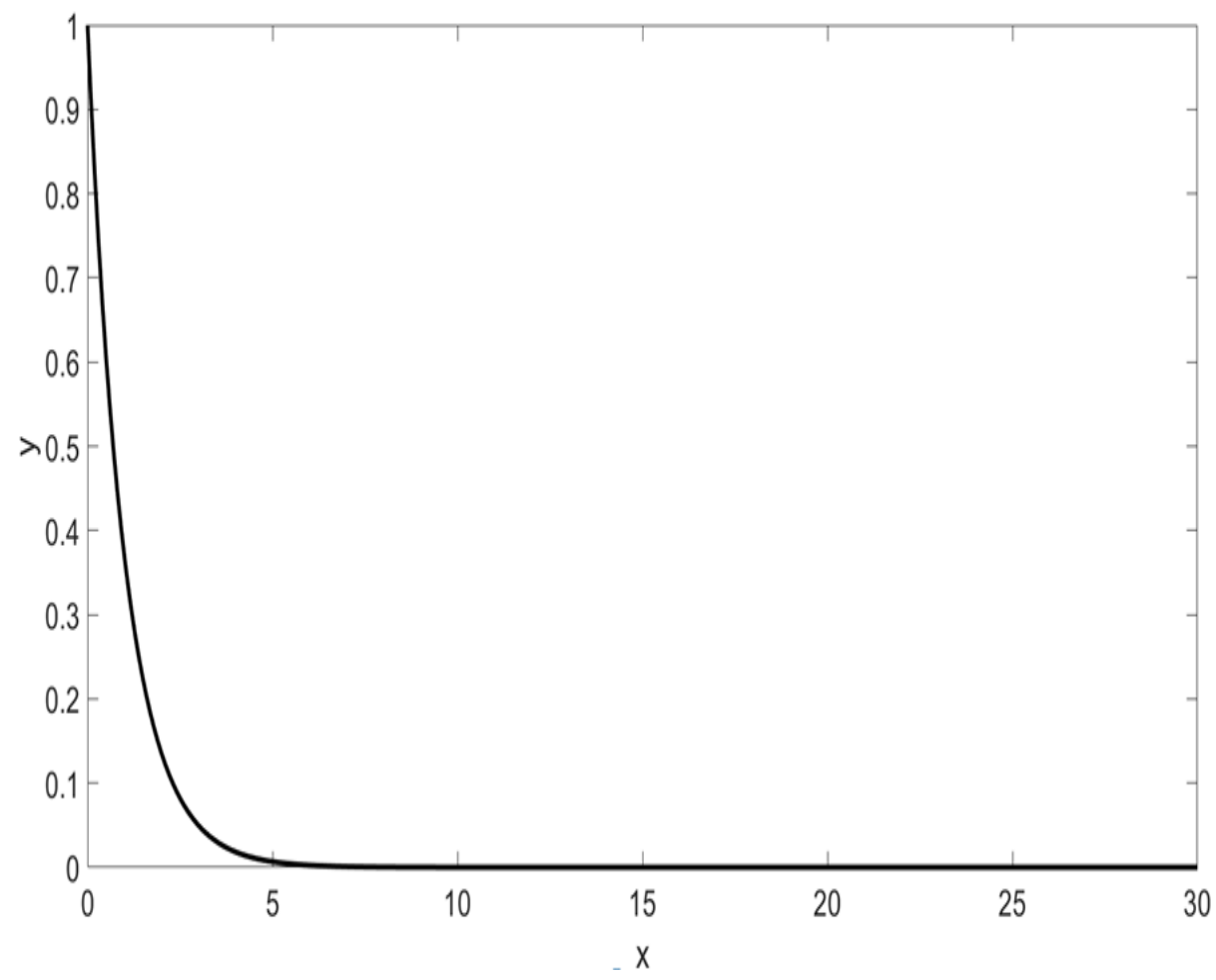

Figure 4 Weibull probability density function for Test product (a: $\mathrm{pH} 4.5$, b: $\mathrm{pH} 6.8$ ).

The plot indicates that the data for generate samples from a log-normal distribution, follows a Weibull distribution. The cumulative distribution (cdf) of the Weibull function it is given by equation 7 :

$p=F((x \mid a, b))=\int_{o}^{x} b a^{-b i b i-1} e-\left(\frac{t}{a}\right)^{b} d t=1-e-\left(\frac{x}{a}\right)^{b}$

The result $p$ is the probability that a single observation from a Weibull distribution with parameters $a$ and $b$ falls in the interval $[0 \mathrm{x}]$. Estimation with $m$-file in MATLAB ${ }^{\mathrm{TM}}$ are graphically presented on Figures 5 and $6: \mathrm{x}=0: 0.1: 30 ; \mathrm{y}=\mathrm{wblcdf}(\mathrm{x}$, $a, b)$ figure; plot(x,y); $\mathrm{x}$-label ('Observation'); $\mathrm{y}$-label ('Cumulative Probability'). The inverse cdf of the Weibull distribution is express as: 
$x=F^{-1}(p \mid a, b)=-a[\ln (1-p)]^{\frac{1}{b}}$

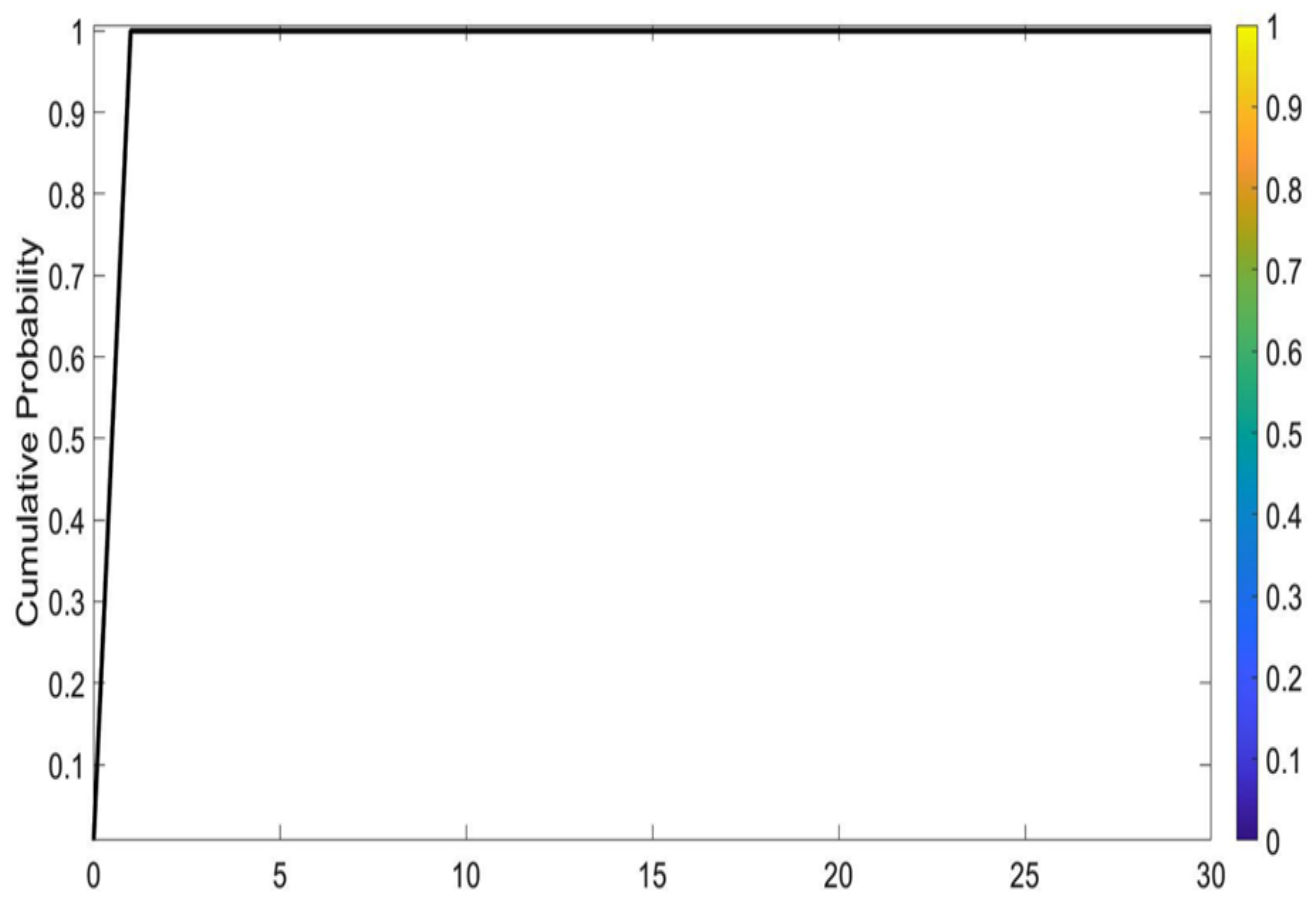

(a)

Observation

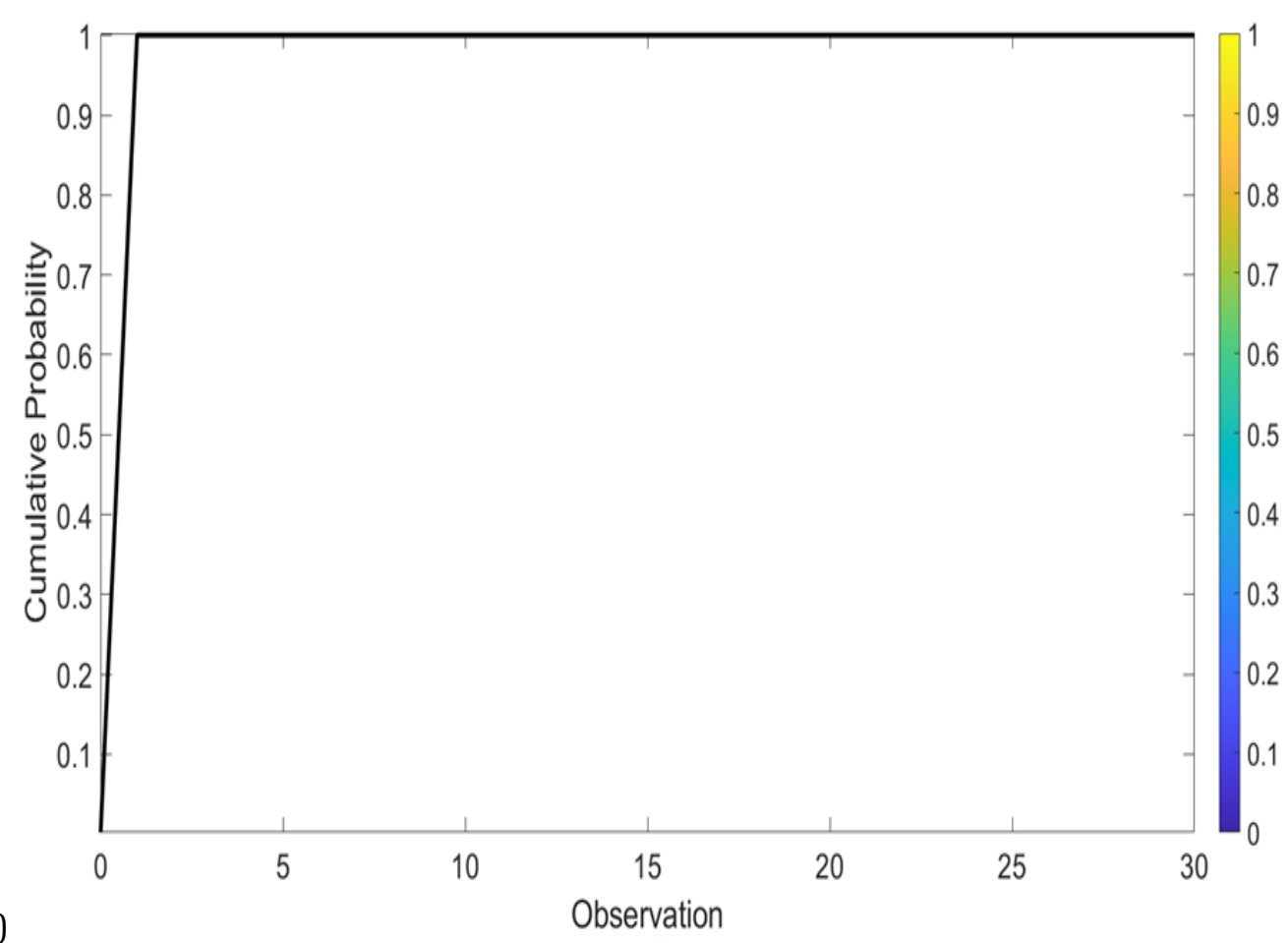

(b)

Figure 5 Weibull cumulative distribution function for Reference product (a: $\mathrm{pH} 4.5$, b: $\mathrm{pH}$ 6.8). 


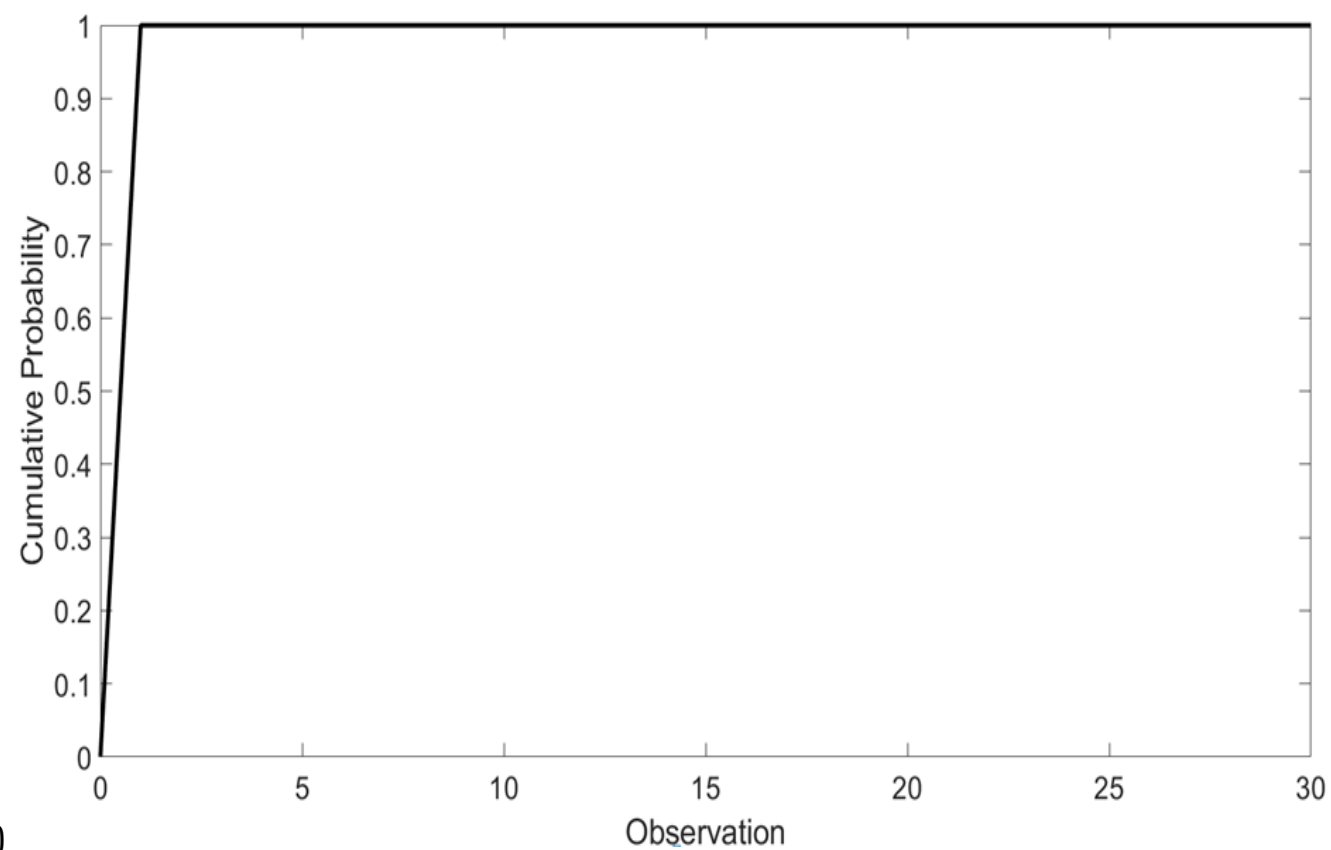

(a)

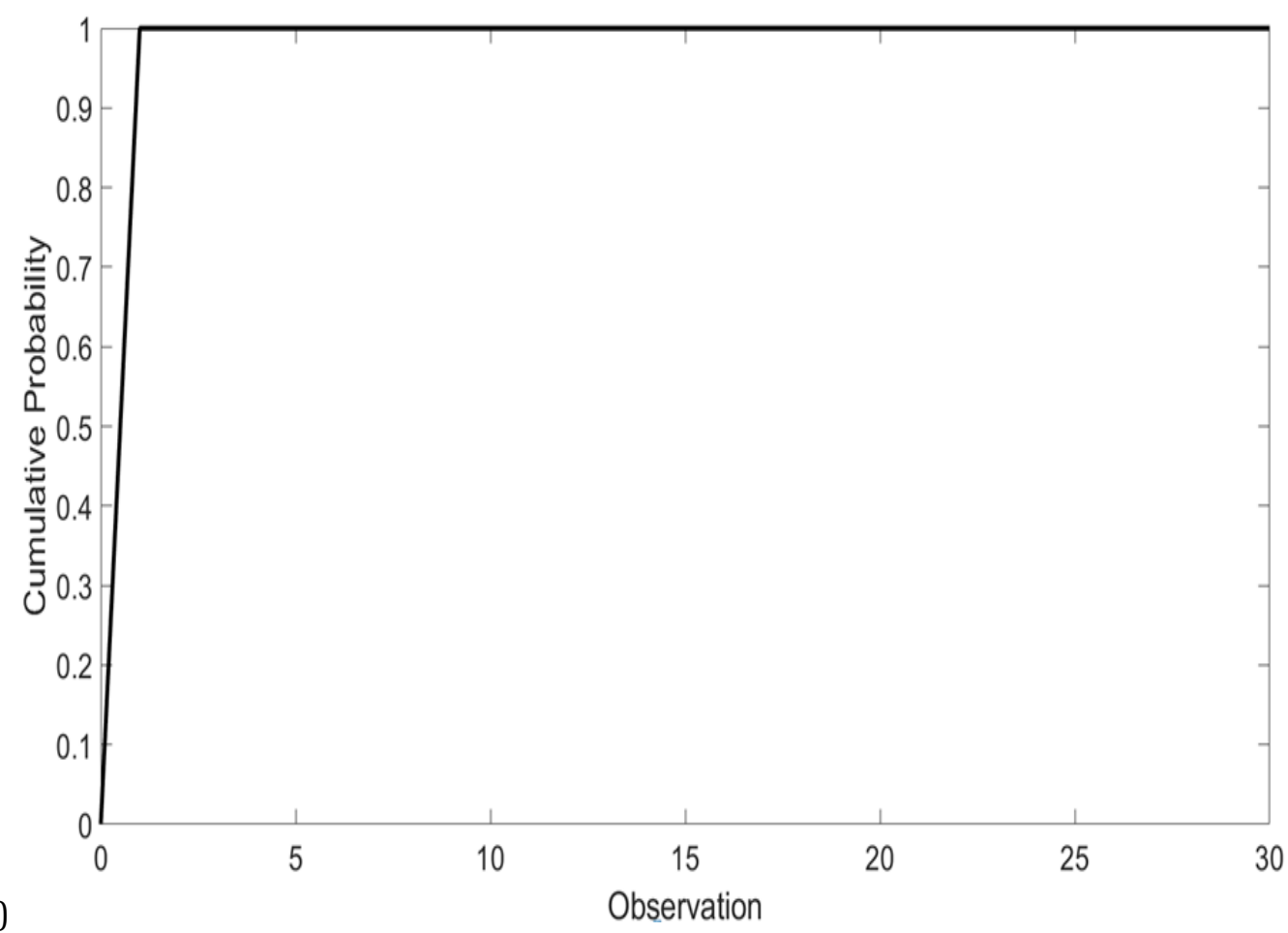

Figure 6 Weibull cumulative distribution function for Test product (a: $\mathrm{pH} 4.5$, b: $\mathrm{pH}$ 6.8).

The result $\mathrm{x}$ is the value where an observation from a Weibull distribution with parameters $a$ and $b$ falls in the range [0 $\mathrm{x}]$ with probability p. In Figures, 7 and 8 the estimates derived for $b$ are plotted $v s$. the estimates for $\mathrm{n}$ for all data analysed. The established linear relationship indicates, not only the mathematical relevance of the exponents $b$ and $n$ (the number of time points tested) of Eq. 7, but also the physical connection of the models parameters and the release mechanism. Evaluation with $m$-file in MATLAB ${ }^{\mathrm{TM}}$ was defined with: $r n g(' d e f a u l t ') \%$ For reproducibility, $\mathrm{r}=$ wblrnd $(\alpha$, $\beta$ ); $\operatorname{wblplot}(\mathrm{r})$. 

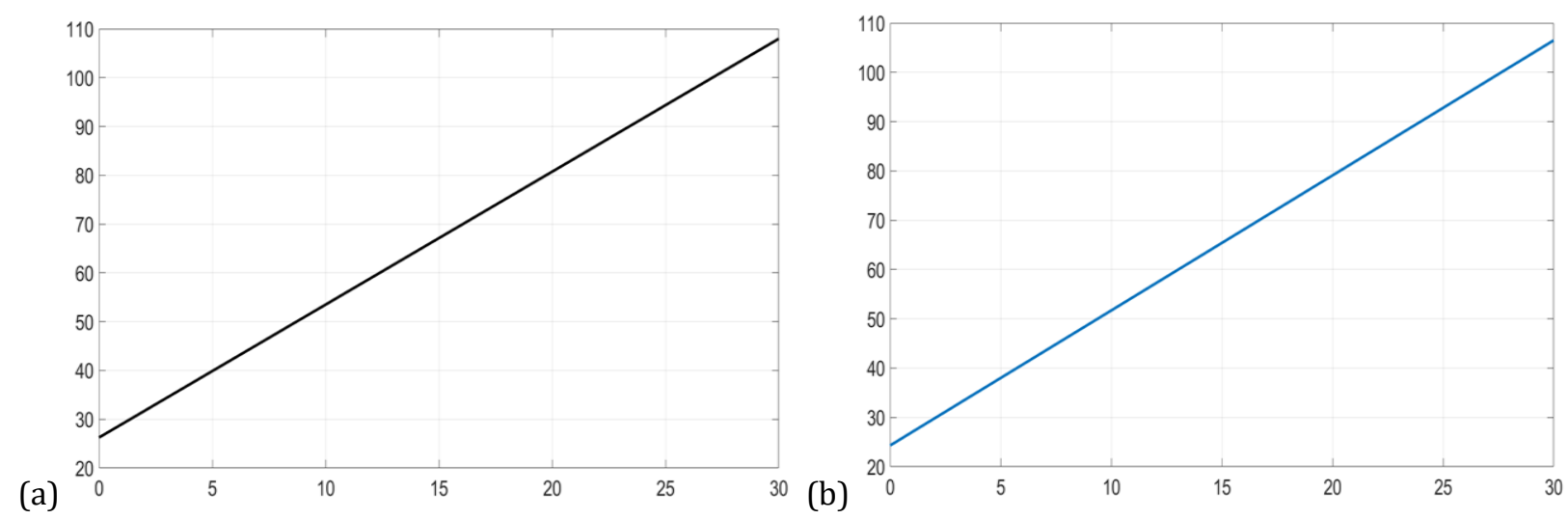

Figure 7 Weibull random numbers for Reference product (a: $\mathrm{pH} 4.5$, b: $\mathrm{pH} 6.8$ ).

(a)
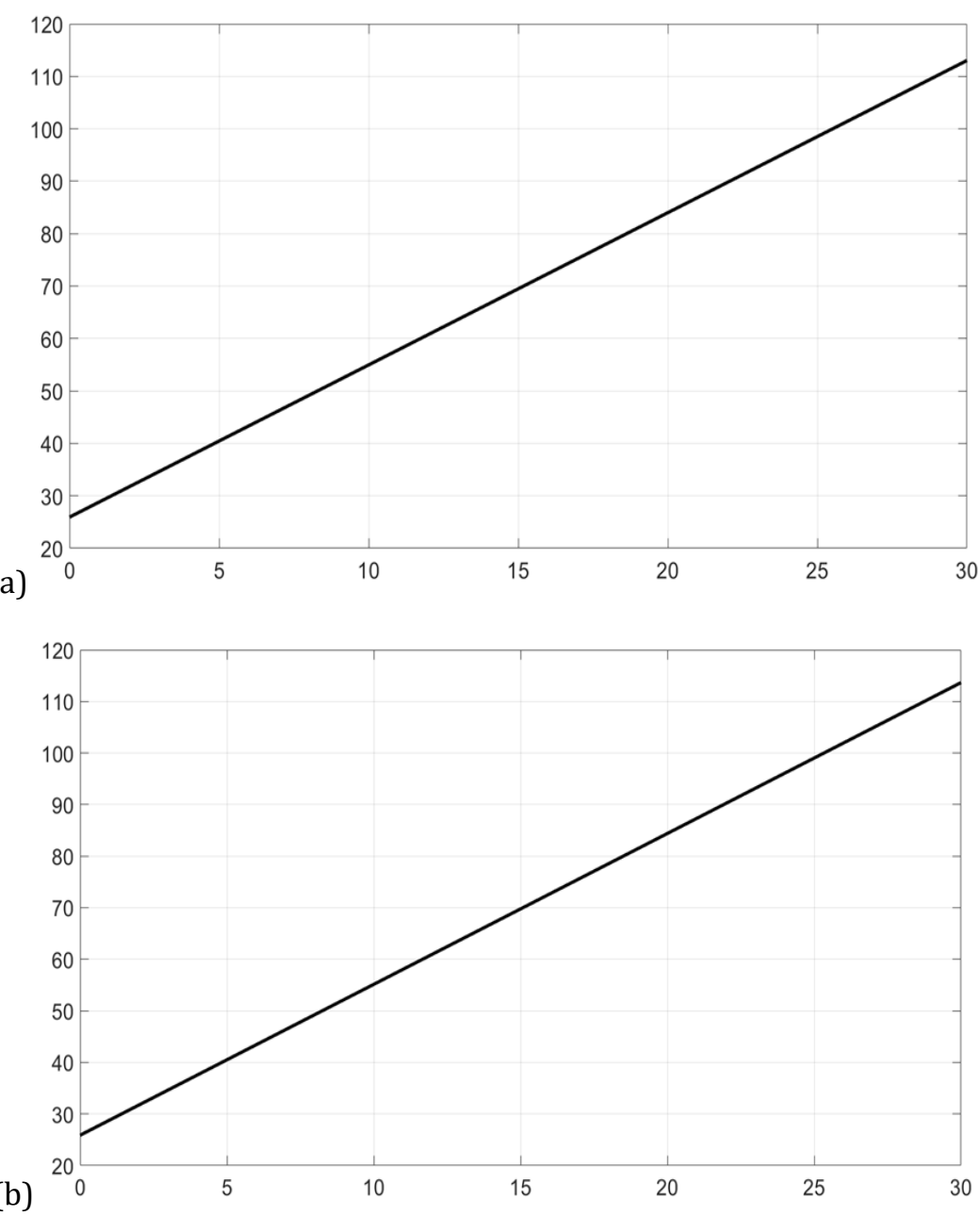

Figure 8 Weibull random numbers for Test product (a: $\mathrm{pH} 4.5, \mathrm{~b}: \mathrm{pH} 6.8$ ).

However, for the inverse function, an accurate estimation for the scale and shape parameters was applied. With the function [wblplot $(\mathrm{x})$ ] in MATLAB ${ }^{\mathrm{TM}}$, intersection point with the $\mathrm{y}$-axis (coefficient $b$ ) was calculated. Further, through expression $\exp (-\mathrm{b} / \beta)$, the parameter $a$ was analysed. In additional, by constructing the frequency histogram from the data, we made an alignment with the probability density of the model distribution. The probability density values of the model distribution are calculated with the values of the integral distribution function within the interval limits of the 
histogram, where $a d x=1$. Usually, the probability density for continuous distribution is calculated as a derivative function $(\mathrm{dP} / \mathrm{dx})[23]$.

Furthermore, the sum of the probabilities were equivalent to one. By normalizing the modified probability density for the values of the twelve (12) samples, each frequency histogram of the model distribution were calculated (Figures 9 and 10) with $m$-file in MATLAB ${ }^{\text {TM: }}$ pd = makedist('Weibull'); rng('default') \% For reproducibility, $r=$ random(pd,100,1); histfit(r,12,'weibull').

(a)
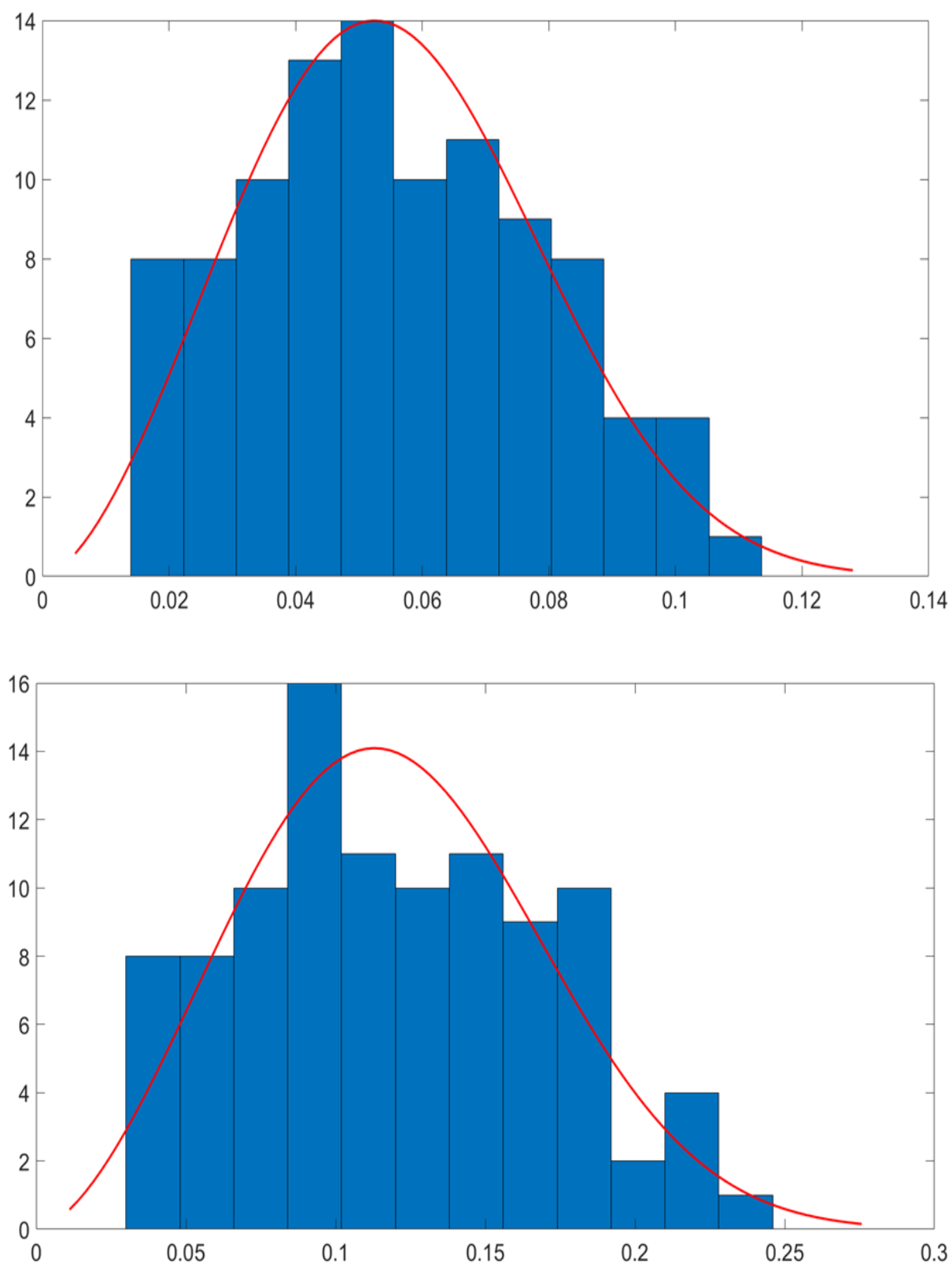

Figure 9 Histogram of the Weibull model distribution for Reference product (a: $\mathrm{pH} 4.5, \mathrm{~b}: \mathrm{pH} 6.8$ ). 
(a)
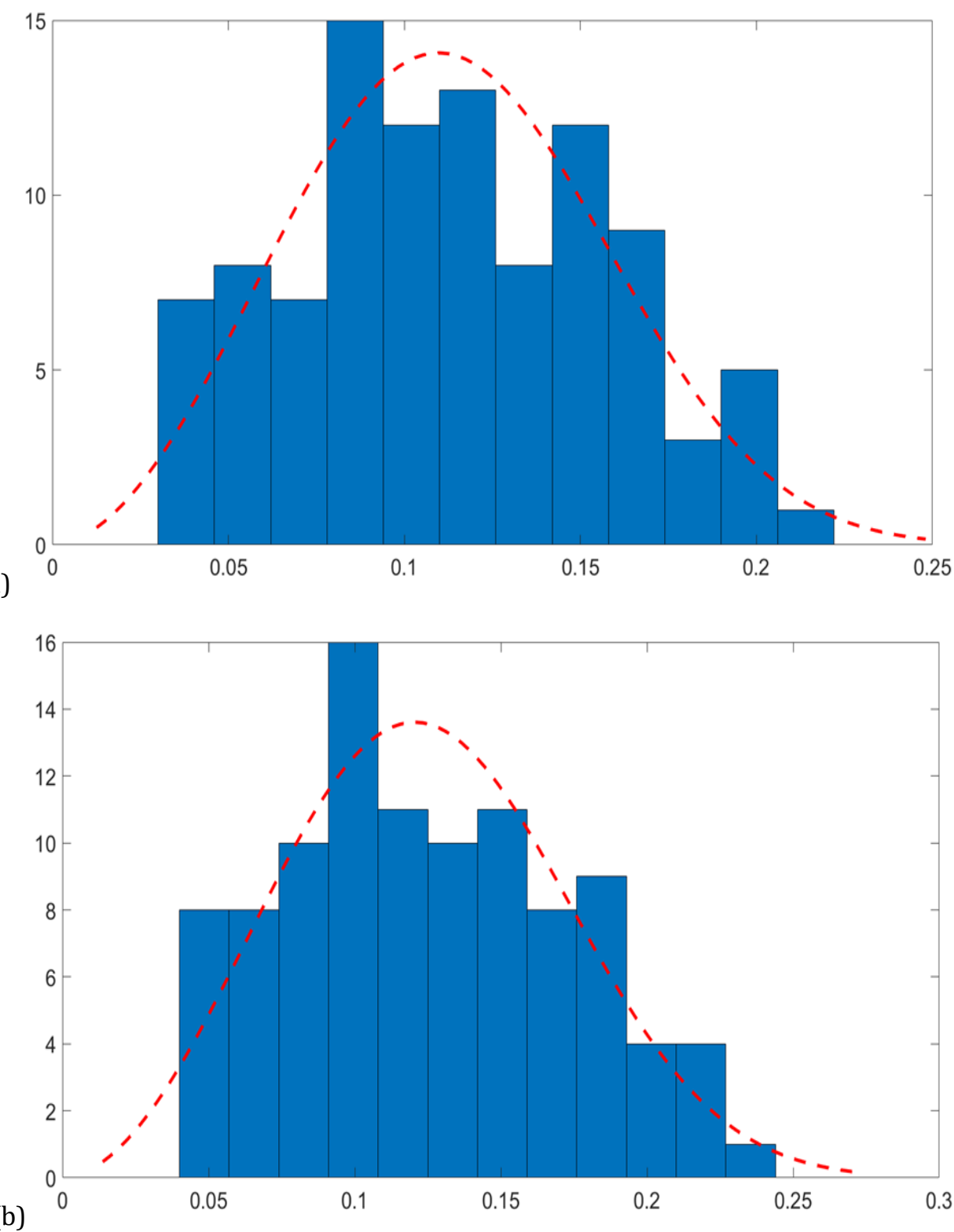

Figure 10 Histogram of the Weibull model distribution for Test product (a: $\mathrm{pH} 4.5, \mathrm{~b}: \mathrm{pH}$ 6.8).

Nevertheless, the hazard function (instantaneous failure rate) was defined as ratio of the pdf and the complement of the cdf. If $f(t)$ and $F(t)$ are the pdf and cdf of a distribution, then the hazard rate is defined as:

$h(t)=\frac{f(t)}{1-F(t)}=\frac{\beta}{\alpha}\left(\frac{t}{\alpha}\right)^{\beta-1}$

It is obvious that $h(t)$ is a decreasing function when $\beta<1$, constant when $\beta=1$, and an increasing function when $\beta>1$. Because of the behaviour of the failure rate function, the Weibull distribution often becomes suitable when the conditions for strict randomness of the exponential distribution are not satisfied, with the shape parameter $\beta$ having a value depending upon the fundamental nature being considered. Therewithal, using the $m$-file in MATLAB ${ }^{\mathrm{TM}}$, hazard function was estimated: $\mathrm{t}=0: .1: 30 ; \mathrm{h} 1=\operatorname{wblpdf}(\mathrm{t}, \alpha, \beta) /(1-\operatorname{wblcdf}(\mathrm{t}, \alpha, \beta)) ; \operatorname{mu}=\operatorname{wblstat}(\alpha, \beta) ; \mathrm{h} 2=\operatorname{exppdf}(\mathrm{t}, \mathrm{mu}) /(1-$ expcdf(t,mu));plot(t,h1,'-',t,h2,"');x label ('Observation'); y label('HazardRate'); legend('Weibull','Exponential', 'location','northwest').

Since, the exponential distribution has a constant hazard function, Weibull distribution is more flexible (Figures 11 and 12). 
World Journal of Advanced Research and Reviews, 2021, 10(01), 056-073

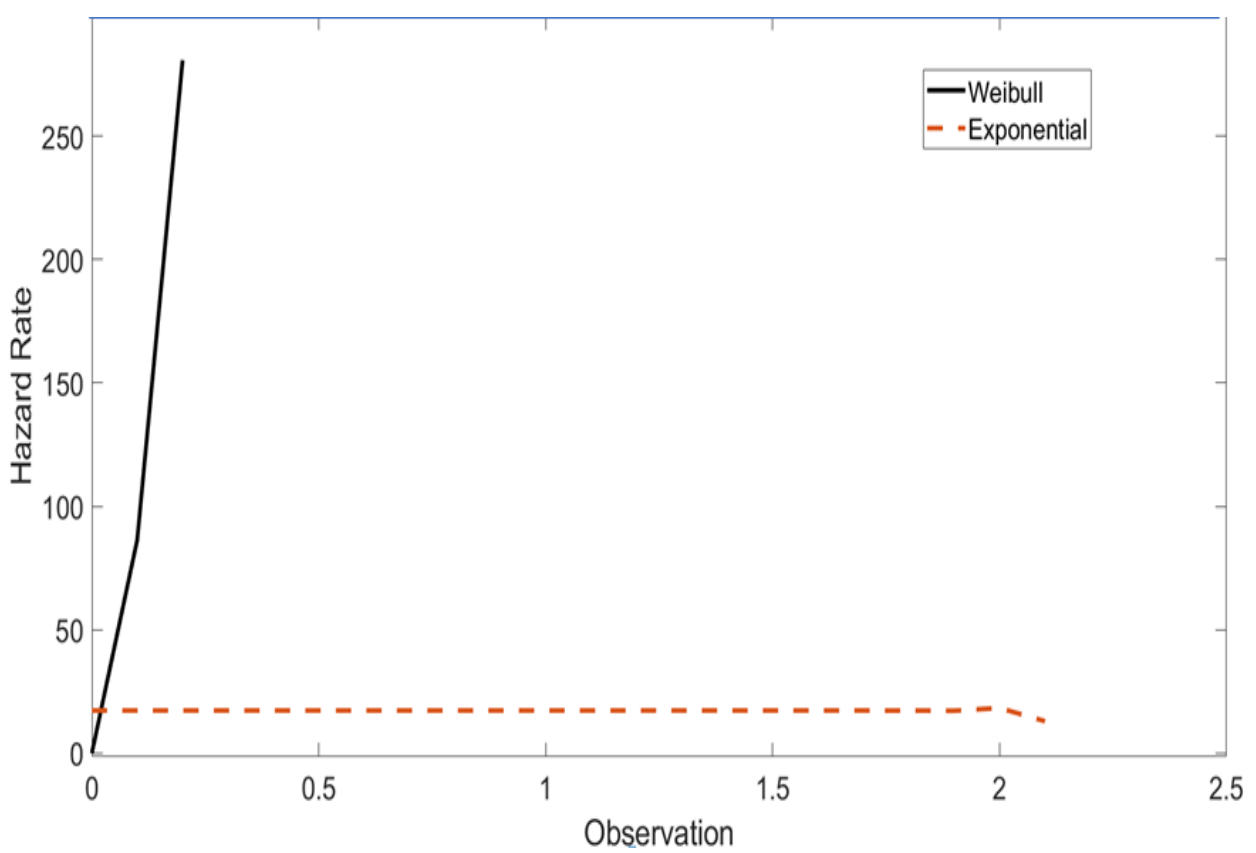

(a)

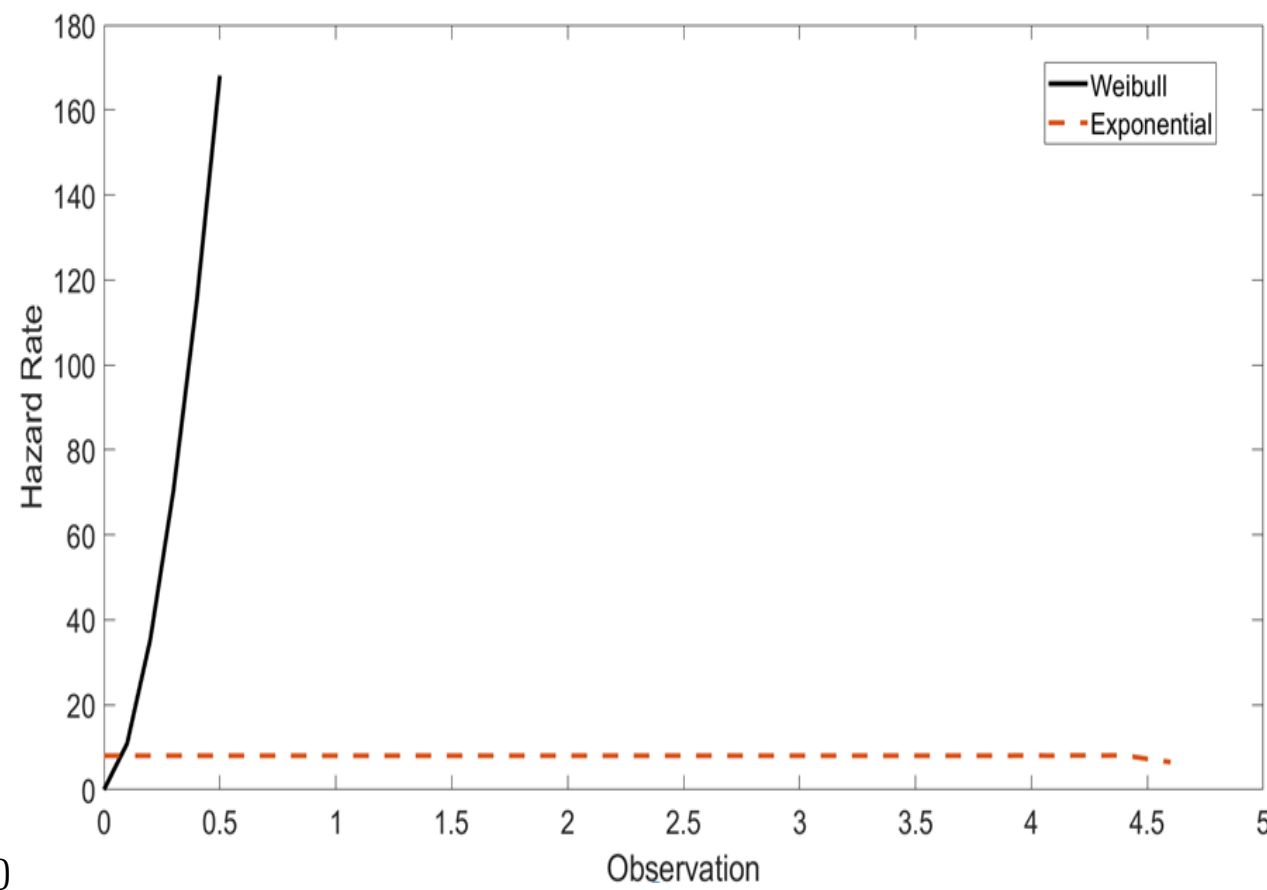

Figure 11 Exponential and Weibull Distribution Hazard Functions for Reference product a: pH 4.5, b: pH 6.8). 


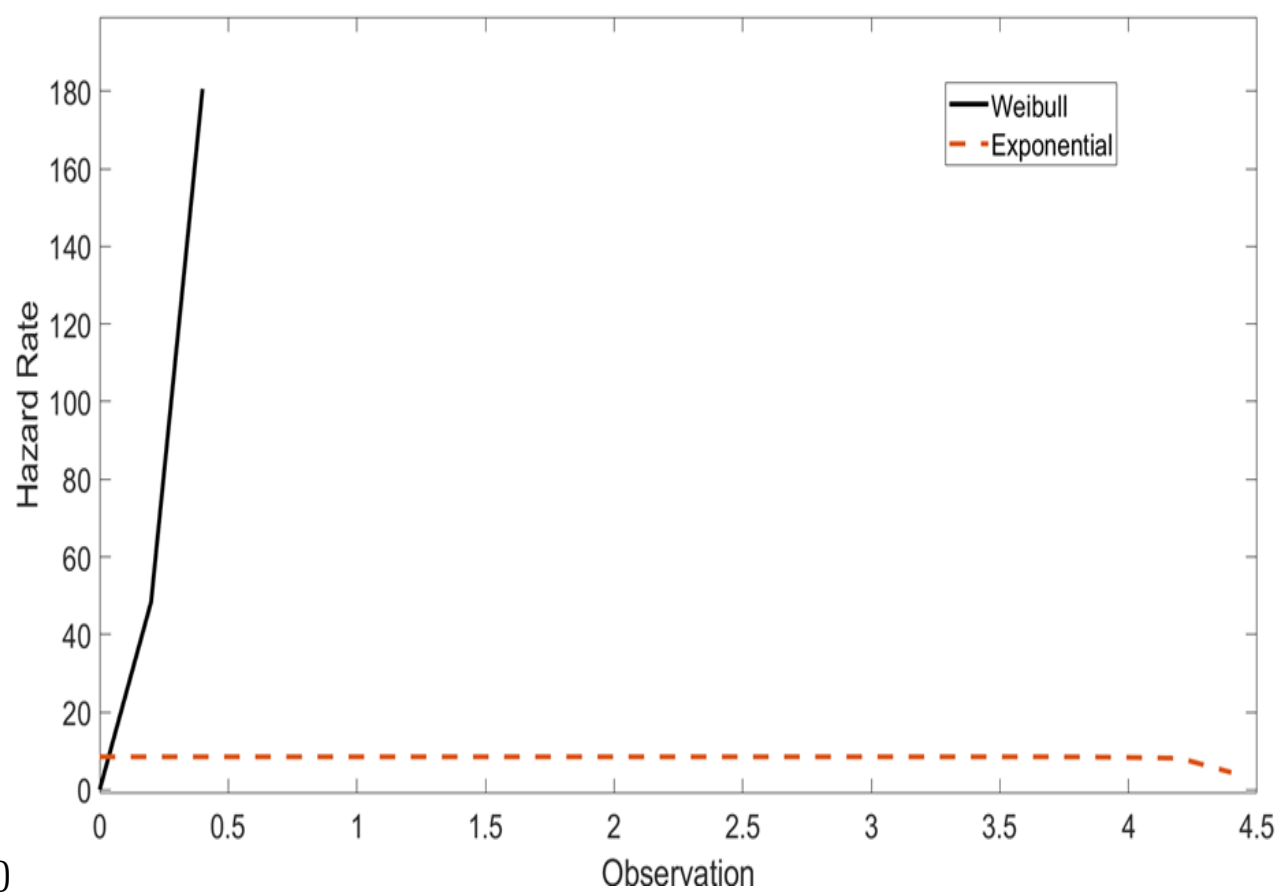

(a)

\section{Observation}

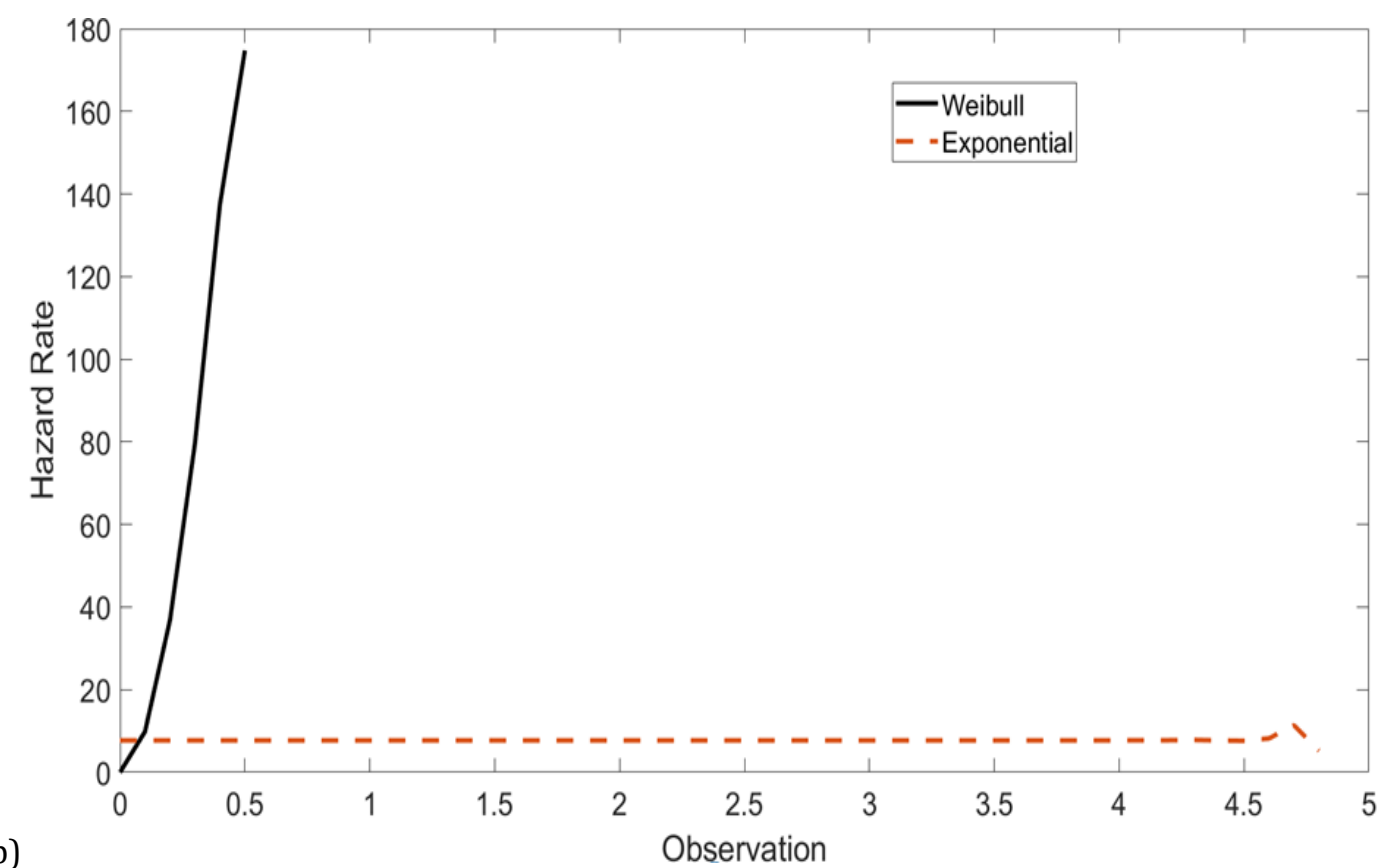

Figure 12 Exponential and Weibull Distribution Hazard Functions for Test product a: $\mathrm{pH} 4.5$, b: pH 6.8).

The data generated with MATLAB ${ }^{\mathrm{TM}}$ software, unequivocally confirmed the similarity between compared in vitro dissolution profiles for the tested products. Simulink is a graphical extension to MATLAB ${ }^{\mathrm{TM}}$ for modelling and simulation of systems. One of the main advantages of Simulink is the ability to model a nonlinear system, which is not possible using a transfer function. Another advantage of Simulink is the ability to take on initial conditions. When a transfer function is built, the initial conditions are assumed to be zero. In the Simulink graphical part, from the MATLABTM

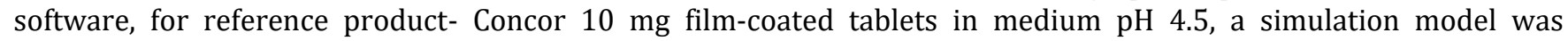
constructed that in the future can replace the classic in vitro experiments for further process parameter optimization. 


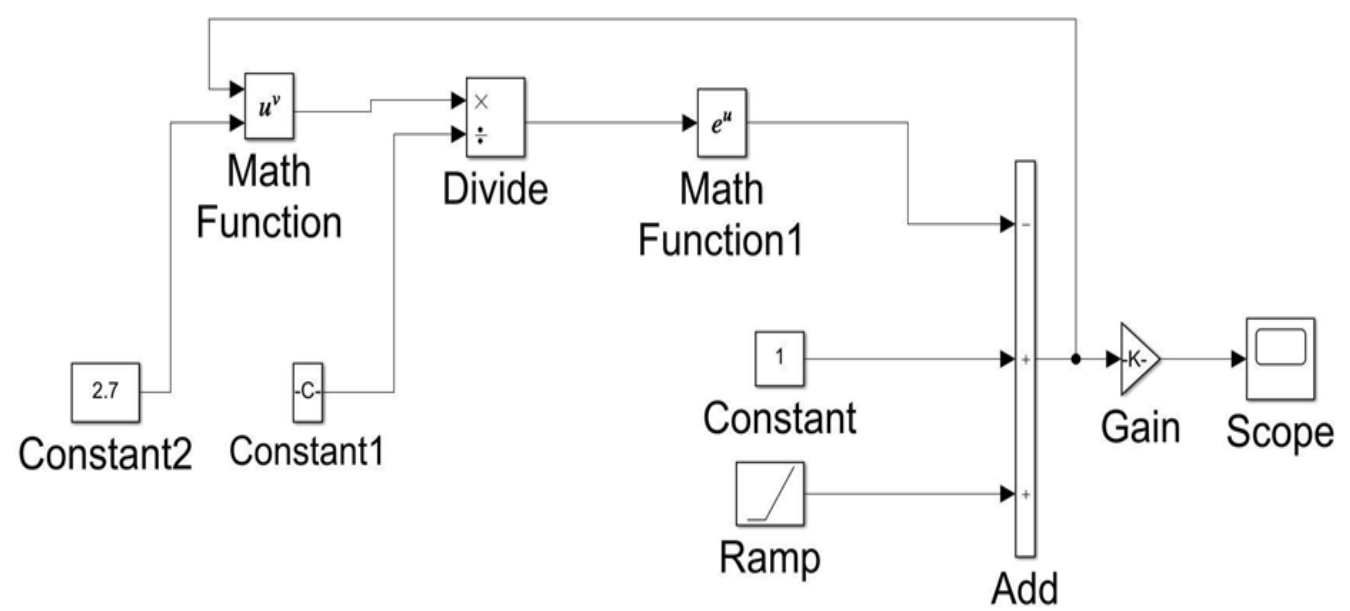

Figure 13 The simulation model of the Weibull distribution for reference product in medium pH 4.5.

Figure 13 shows the simulation model (imitation model) that can be used to analyse the Weibull distribution function for reference product (Concor $10 \mathrm{mg}$ film-coated tablets) in medium $\mathrm{pH} 4.5$, with linear equation $y=2.9031 \mathrm{x}+25.936$, and values for shape parameters $\beta=2.7$ and scale parameter $\alpha=0.000065$. Figure 14 shows the simulation diagram of the mathematical model for the Weibull distribution. The diagram shows that in the $15^{\text {th }}$ minute the release of the active substance from the formulation will be below $85 \%$.

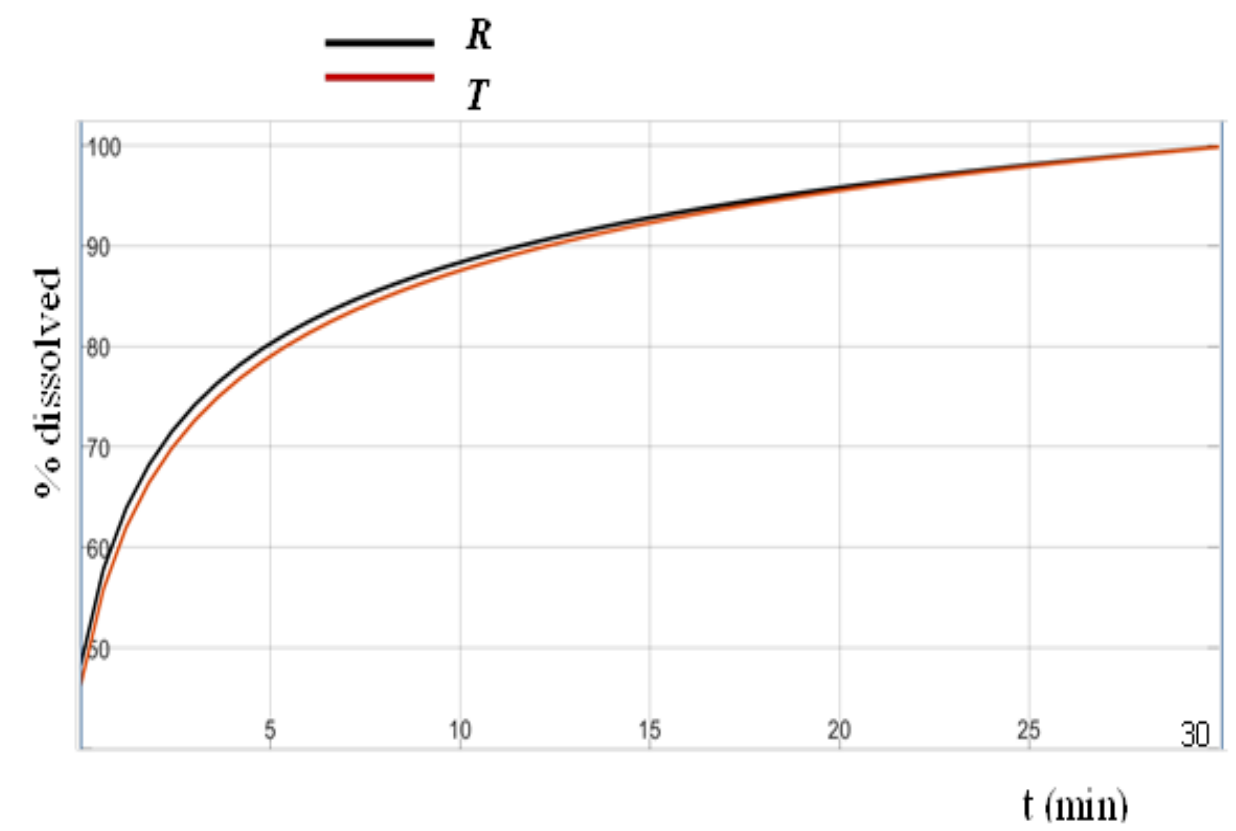

Figure 14 Diagram of the simulation model for the Weibull distribution for Reference and Test products in medium pH 4.5.

As well, from the Simulink model the parameter $\beta$ and the slope of the line with respect to the $\mathrm{x}$-axis can be determined. With the MATLAB ${ }^{\mathrm{TM}}$ / Simulink software package, the parameters of the Weibull function can be assumed, calculated and the results further validated. Thus, the requirements of the $V$-development model for the development of new technologies and processes are met (Figure 15).

Model-Based Design is a process that enables more effective development of dynamic systems, such as dissolution testing. At the core of the V-development model is process simulation and modelling. Imitation is a method to make an optimal solution and gives us the opportunity to evaluate the quality of the system (process) in relation to another. The term simulation means an experimental approach to defining the functional properties and capabilities of the basic model through its imitation model. In our case, the basic model was represented by the Weibull function. 


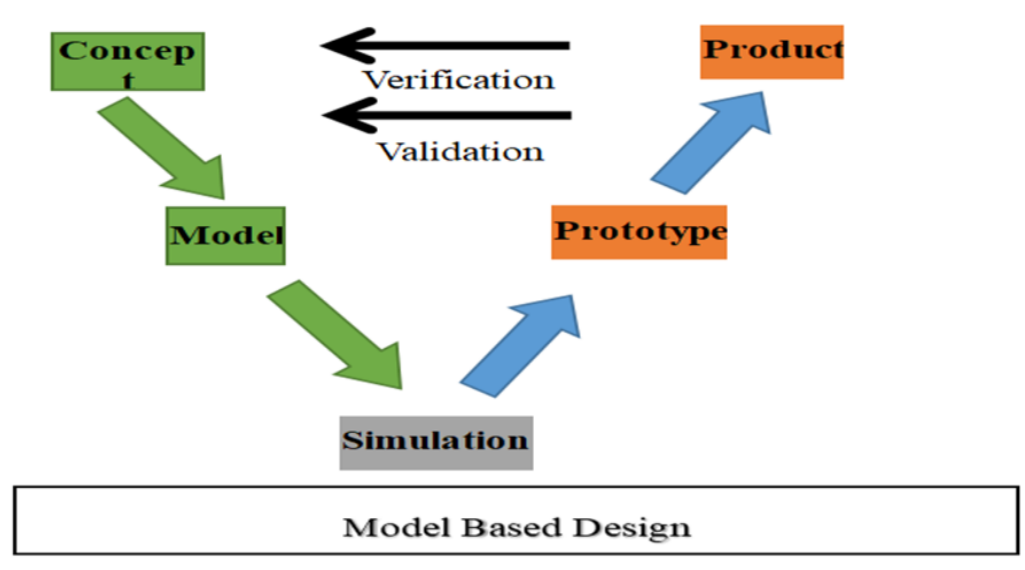

Figure 15 V-development model

The reliability of generic medicines depends on the development and manufacture decisions made before the marketing authorization phase, proves to be safe and effective as the reference formulation, which in turn affects successes when the product is placed in the post-approval phase. Despite this, we have proposed a new principal component analysisbased approach for testing "similarity" of active substance dissolution profiles. This was mainly due to the facts that the Weibull non-linear effects model is more discriminating, taking into account data variability within the reference lot in order to build the confidence ellipses. Variations within the test lot, as well as shape and size of the dissolution curves have also influence on the conclusions. It should be highlight that, the used simulations had advantages in order to shorten the period required for the development; systematic approach and guideline for further manufacturer process improvement, as well as beneficial optimization in terms of in vitro behaviour of the generic product. However, the advantages of the Simulink model are to simulate the actual performance that set out as a tool for further design, optimization or evaluation purposes. This strategy opens a door for implementation of a new methodology for comparing in vitro dissolution profiles, through precise calculation and analysis of the obtained data in the MATLAB ${ }^{\mathrm{TM}}$ / Simulink software that contains the Weibull function in its structure.

\section{Conclusion}

Prediction of the in vivo performance of the medicinal product from the in vitro studies is the major challenging job for the pharmaceutical industries. In this study, application of MATLAB ${ }^{\mathrm{TM}}$ simulation software was used for accurate prediction of in vivo behaviour of the active substance, which will provide additional support in generic medicine development.

The evaluation of the generic-test product and comparison with the reference product, was performed using the in vitro dissolution rate test, which is expected to reflect the performance and quality according to the reference product. Evaluation of the kinetics of dissolution data using MATLAB ${ }^{\mathrm{TM}}$ simulation software indicate mainly fitting to Weibull non-linear model. It is considered that this approach have the benefit of a better prediction of responses outside the range of observed data. An attendant advantage, in the cases studied here, is that sample runs of the program demonstrated that the results were satisfactory, and MATLAB ${ }^{\mathrm{TM}}$ simulation software could be served as a useful tool for dissolution data analysis and to fit all kinds of dissolution curves with sufficient accuracy. MATLAB ${ }^{\mathrm{TM}}$ simulation software is capable of performing the existing techniques for comparing active substance release data.

\section{Compliance with ethical standards}

\section{Acknowledgments}

The authors would like to thank Prof. Dr. Ivan Mickoski, Faculty of Mechanical Engineering, Skopje, R. North Macedonia for his initial input on this work and his kind support for the publication of the data from this research project.

\section{Disclosure of conflict of interest}

The authors declare that there is no conflict of interest. 


\section{Statement of informed consent}

"Informed consent was obtained from all individual participants included in the study."

\section{References}

[1] Siepmann J, Siepmann F. Mathematical modeling of drug dissolution. Int J Pharm. 2013; 453(1): 12-24.

[2] Cost P, Sousa Lobo JM. Modelling and comparison of dissolution profiles. Eur J Pharm Sci. 2001; 13(2): 123-33.

[3] Vranić E, Mehmedagić A, Hadzović S. Mathematical methods for quantification and comparison of dissolution testing data. Bosn J Basic Med Sci. 2002; 2(1-2): 49-52.

[4] Sathe PM, Tsong Y, Shah VP. In-Vitro Dissolution Profile Comparison: Statistics and Analysis, Model Dependent Approach. Pharmaceutical Research. 1997; 13(12): 1799-1803.

[5] Langenbucher F. Linearization of dissolution rate curves by the Weibull distribution. J Pharm Pharmacol. 1972; 24(12): 979-81.

[6] Dokoumetzidis A, Kalantzi L, Fotaki N. Predictive models for oral drug absorption: From in silico methods to integrated dynamical models. Expert Opinion on Drug Metabolism \& Toxicology. 2007; 3(4): 491-505.

[7] European Pharmacopoeia 10 th Edition. Recommendations on dissolution testing. 5.17.1; 2020.

[8] European Pharmacopoeia 10 $10^{\text {th }}$ Edition. Dissolution Test for Solid Dosage Forms. 2.9.3; 2020.

[9] US Pharmacopoeia, 2019. Monograph for Hydrochlorothiazide and Bisorprolol Tablets. USP-42- NF 37.

[10] Polli JE, Rekhi GS, Shah VP. Methods to compare dissolution profiles. Drug Inf. J. 1996; 1113-1120.

[11] Polli JE, Rekhi GS, Augsburger LL, Shah VP. Methods to compare dissolution profiles and a rationale for wide dissolution specifications for metoprolol tartrate tablets. J. Pharm. Sci. 1997; 86(6): 690-700.

[12] Murthy DNP, Xie M, Jiang R. Weibull Models. Wiley, New York. 2003.

[13] Weibull W. A statistical distribution function of wide applicability. J. Appl. Mech. 1951; 18: 293-296.

[14] Lai CD, Xie, M, Murthy DNP. Modified Weibull model. IEEE Trans. Reliab. 2003; 52(1): 33-37.

[15] Crowder MJ, Kimber A, Sweeting T, Smith R. Statistical Analysis of Reliability Data. Reprinted. London: Chapman \& Hall. 1995.

[16] Devroye L. Non-Uniform Random Variate Generation. New York, NY: Springer New York. 1986.

[17] Evans M, Hastings N, Peacock B. Statistical Distributions. 2nded. New York: J. Wiley. 1993.

[18] Lawless, Jerald F. Statistical Models and Methods for Lifetime Data. 2nd ed.Wiley Series in Probability and Statistics. Hoboken, N.J: Wiley-Interscience. 2003.

[19] Meeker, William Q, Luis AE. Statistical Methods for Reliability Data. Wiley Series in Probability and Statistics. Applied Probability and Statistics Section. New York: Wiley. 1998.

[20] European Medicines Agency, EMEA Guideline on the investigation of bioequivalence (CPMP/EWP/QWP/1401/98 Rev. 1/ Corr), London. 2010.

[21] FDA Guidance for industry: Dissolution testing of immediate release solid oral dosage forms, In. Rockville, MD. 1997.

[22] Sathe PM, Tsong Y, Shah VP. In-vitro dissolution profile comparison: statistics and analysis, model dependent approach. Pharm. Res. 1996; 13: 1799-803. 